\title{
Depth Control for an Over-Actuated, Hover-Capable Autonomous Underwater Vehicle with Experimental Verification
}

\author{
Kantapon Tanakitkorn ${ }^{\mathrm{a}, \mathrm{c}, 1}$, Philip A. Wilson ${ }^{\mathrm{a}, 2}$, Stephen R. Turnock ${ }^{\mathrm{a}, 3}$, Alexander B. Phillips ${ }^{\mathrm{b}, 4}$ \\ ${ }^{a}$ Fluid Structure Interactions Research Group, University of Southampton, UK \\ ${ }^{b}$ Marine Autonomous Robotic Systems, National Oceanography Centre, Southampton, UK \\ ${ }^{c}$ This project is sponsored by the Faculty of International Maritime Studies, Kasetsart University, SriRacha Campus.
}

\begin{abstract}
A PI-D based control system is developed for over-actuated, hover-capable AUVs which enables a smooth transition from hover-style to flight-style operation with system stability and convergence proven using a Lyapunov approach. The performance of the controller is demonstrated by simulation and is verified through experiments. The approach is able to operate over a range of vehicle ballasting configurations, and to imposed external disturbances. The proposed system is computationally inexpensive and does not require a detailed hydrodynamic model to implement. By monitoring the energy consumption on board, the cost of maintaining depth at a range of forward speeds with different buoyancy conditions can be quantified, and their impact on the cost of transport is highlighted for future optimisation of energy consumption.
\end{abstract}

Keywords: Delphin2; AUV; depth control; over-actuated; hover-capable; energy consumption.

\section{Introduction}

Autonomous underwater vehicles (AUVs) are normally designed with an emphasis on minimising total resistance to suit long-range flight-style operation — they are of a torpedo-shaped body that is equipped with a main propeller and control surfaces $[1,2]$. These conventional AUVs, however, are not able to carry out detailed inspection tasks at zero or slow forward speeds since the control surfaces become ineffective in this regime [3, p.169].

Various concepts for next generation of hovercapable AUVs have been developed to overcome such a limitation $[4,5,6,7]$. Typically, thrusters are added to the design to provide additional control forces in low-speed operation, as illustrated

\footnotetext{
${ }^{1}$ K.Tanakitkorn@soton.ac.uk

${ }^{2}$ Philip.Wilson@soton.ac.uk

${ }^{3}$ S.R.Turnock@soton.ac.uk

4 abp@noc.co.uk
}

in figure 1. Since this class of the AUVs can be considered to have a redundant set of actuators for controlling a given degree of freedom, they are referred to as over-actuated AUVs.

Different control strategies are required for controlling an over-actuated AUV that is operating in different styles. For flight-style operation, stern planes are used to adjust the pitch angle of the vehicle which in turn results in depth changes. A controller for flight-style operation may be designed based on a cascaded depth and pitch control structure $[8,9,10,11,12]$. In contrast, the control problem for hover-style operation may be considered as two parts $[13,14,15]$. First, a control law that determines the generalised forces required. Second, a control allocation that distributes the control forces onto vertical thrusters and stern planes. Such an approach has been implemented by [16, 17].

Alternatively, the vehicle depth, in hover-style operation, may also be controlled by adjusting 
its displacement via a buoyancy engine [18]. Although this approach consumes less energy compared to the thruster operations, the mechanism is typically slow [19] and additional tunnel thrusters are still required for fast vehicle response. A moving mass actuator may be used to trim the vehicle [20]. This can extend the flightstyle control in the low-speed regime without requiring additional forces from the thrusters; however, this approach cannot be used at zero speed. A more radical approach which requires a significant increase in system complexity is the addition of internal control moment actuators that can alter altitude even at zero speed [21].

Simulation studies on a concept of a unified control system for over-actuated AUVs are presented in $[22,23]$. Weighting functions are used to provide a seamless transition between hoverstyle and flight-style strategies.

Model predictive control (MPC) has also been applied to depth control of over-actuated AUVs [24]. This has been shown to provide acceptable results over a range of speeds but is computationally expensive due to the need to solve an optimisation problem before each control allocation. Alternatively, sliding mode control (SMC) technique can also be used [25, 26]. A downside of this approach is that it is susceptible to chatter; a range of approaches has been developed to overcome this $[27,28]$.

This paper focuses on design, simulation and experimental testing of proportional-integralderivative (PID) based diving control system for over-actuated AUVs that can be used over a wide range of operating speeds with a seamless transition between hover-style and flight-style control.

The paper is organised in a following order. The Delphin2 AUV is presented in section 2, followed with a mathematical model in section 3 . The proposed control system is explained in section 4. Section 5 provides a stability and convergence analysis. Simulation and experimental setup are explained in section 6 and 7 respectively. Results are discussed in section 8. This follows with a conclusion in section 9 .

\section{Delphin2 AUV}

Delphin2 is a torpedo-shaped AUV with a length of $1.96 \mathrm{~m}$ and mid-body diameter of $0.26 \mathrm{~m}$ [29]. The AUV is equipped with a main rear propeller, four rear control surfaces in a cruciform arrangement. It is also fitted with four throughbody tunnel thrusters: two horizontal and two vertical. (See figure 1.) Hence, it can effectively perform a wide range of missions, ranging from a zero-speed hovering to longer range flight-style survey missions at a maximum forward speed of approximately $1 \mathrm{~m} / \mathrm{s}$.

It is typical for an AUV to be slightly positively buoyant [30, p.89], allowing the vehicle to naturally return to the water surface in the event of system failure. Due to this, Delphin2 is typically ballasted to be $6 \mathrm{~N}$ positively buoyant.

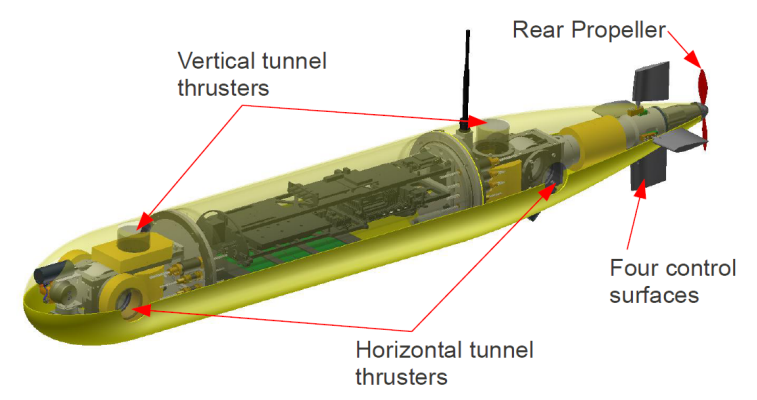

Figure 1: Delphin2 AUV [31].

To conduct this work, significant upgrades have been made to the vehicle that is described in [29]; these include:

- The CPU was replaced with an Intel Atom D525 dual-core processor $1.8 \mathrm{GHz}$, reducing the hotel load from $50 \mathrm{~W}$ to $30 \mathrm{~W}$ and enhancing endurance.

- The OceanServer Compass and rate-gyro were replaced by Xsens $4^{\text {th }}$ generation MTi30 IMU, providing higher precision navigation performance.

- The 6-channel TSL motor control board was replaced by four identical maxon motor control 1-Q-EC Amplifier DECS 50/5. These reduced the tunnel thruster deadband from $450 \mathrm{rpm}$ to $150 \mathrm{rpm}$, enhancing hover performance. 
- A power monitoring system has been developed: it is composed of Arduino Nano, and voltage and current sensors. This allows power consumption of the actuators to be continuously recorded.

\section{Vehicle Dynamics Modelling}

The kinematics and dynamics model are developed based on Delphin2 dynamics according to the SNAME [32] notation. The two coordinate systems used in the model are illustrated in figure 2 . The first is a body-fixed frame (b-frame) that is fixed to the AUV body and aligned with the longitudinal axis. The second is an earth-fixed coordinate system where $x$-, $y$ - and $z$-axis point northward, eastward and downward respectively; hence the name $N E D$-frame.

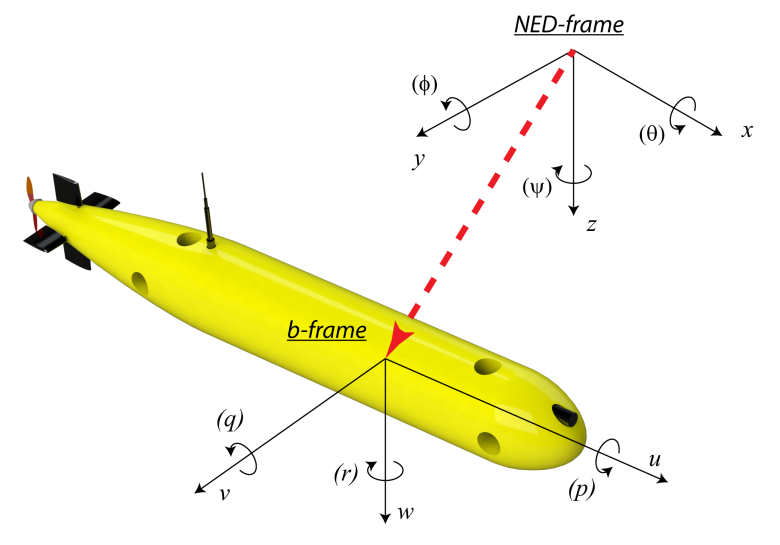

Figure 2: Coordinate systems.

Rigid-body parameters of Delphin2 AUV are obtained partly from a direct measurement and partly from a 3D CAD model. Most of the hydrodynamic derivatives used are estimated from planar motion mechanism (PMM) and rotating arm experiments performed on a torpedo-shaped AUV with a similar shape [33]. Surge damping coefficient and control surfaces derivatives are inferred from wind tunnel tests on Delphin2 [34]. A quadratic heave damping coefficient is estimated from a free ascent test, see Appendix A.

These model parameters are detailed in Appendix B.

\subsection{DOF Equations of Motion}

The vertical plane AUV model may be described in a matrix form [26]:

$$
\boldsymbol{M} \cdot \dot{\nu}+\boldsymbol{C}(\nu) \cdot \nu+\boldsymbol{D}(\nu) \cdot \nu+g(\eta)=\tau+\Delta \tau,
$$

where $\nu=[u, w, q]^{T}$ is the velocity vector, $\eta=$ $[x, z, \theta]^{T}$ is a pose vector and $\tau=[X, Z, M]^{T}$ is a generalised force vector; $\Delta \tau$ represents a factor that is considered in this work but is missing from [26] — this is explained later in this section. The inertia matrix, Coriolis-centripetal matrix, damping matrix and hydrostatic force vector for decoupled surge dynamics are:

$$
\begin{gathered}
\boldsymbol{M}=\left[\begin{array}{ccc}
m-X_{\dot{u}} & 0 & 0 \\
0 & m-Z_{\dot{w}} & 0 \\
0 & 0 & I_{y y}-M_{\dot{q}}
\end{array}\right] \\
C(\nu)=\left[\begin{array}{ccc}
0 & 0 & c_{1,3} \\
0 & 0 & c_{2,3} \\
c_{3,1} & c_{3,2} & 0
\end{array}\right]
\end{gathered}
$$

where $c_{1,3}=-c_{3,1}=-m\left(x_{g} q-w\right)-Z_{\dot{w}} w$ and $c_{2,3}=-c_{3,2}=-m\left(z_{g} q+u\right)+X_{\dot{u}} u$,

$\boldsymbol{D}(\nu)=-\left[\begin{array}{ccc}X_{u}+X_{|u| u \mid}|u| & 0 & 0 \\ 0 & Z_{w}+Z_{|w| w \mid}|w| & Z_{q}+Z_{|q| q}|q| \\ 0 & M_{w}+M_{|w| w \mid}|w| & M_{q}+M_{|q| q \mid}|q|\end{array}\right]$

$$
g(\eta)=\left[\begin{array}{c}
(W-B) \sin (\theta) \\
-(W-B) \cos (\theta) \\
\left(z_{g} W-z_{b} B\right) \sin (\theta)+\left(x_{g} W-x_{b} B\right) \cos (\theta)
\end{array}\right]
$$

The origin of the $b$-frame is chosen to be coincident with the centre of buoyancy $r_{b}=\left[x_{b}, y_{b}, z_{g}\right]$. The centre of gravity $r_{g}=\left[x_{g}, y_{g}, z_{g}\right]$ is just below $r_{b}$.

A transformation matrix $\boldsymbol{J}(\eta)$ is used to correlate the velocity vector $\nu$ in $b$-frame to the velocity vector $\dot{\eta}$ in $N E D$-frame under the assumption of zero heel angle:

$$
\begin{gathered}
\dot{\eta}=\boldsymbol{J}(\eta) \nu \\
\boldsymbol{J}(\eta)=\left[\begin{array}{ccc}
\cos (\theta) & \sin (\theta) & 0 \\
-\sin (\theta) & \cos (\theta) & 0 \\
0 & 0 & 1
\end{array}\right] .
\end{gathered}
$$




\subsection{Drag from Tunnel Thruster Operation}

When a through-body tunnel thruster is operating, it sucks water into one side of the tunnel and ejects a jet of water on the opposite side. This water jet may be considered as an increasing in the effective frontal area of the vehicle, increasing the vehicle drag. This effect is most pronounced when the jet is strong relative to the ambient flow.

Palmer presents an extensive study of a tunnel thrusters performance on a torpedo-shaped AUV with a similar shape and size which utilised the same thruster units as Delphin2 [35]. The experimental result in figure 3 suggests that a change in volumetric surge damping coefficient may be modelled as a function of the ratio between surge speed $(u)$ and jet speed $\left(u_{j}\right)$ :

$$
\begin{aligned}
\Delta C_{d, t h}= & 0.5660 \cdot e^{\left(-7.6089\left(\frac{u}{u_{j}}\right)\right)} \\
& +0.0565 \cdot e^{\left(-0.8968\left(\frac{u}{u_{j}}\right)\right)},
\end{aligned}
$$

where thruster jet speed is given by $u_{j}=$ $\sqrt{4 F_{t h} /\left(\rho \pi D_{t h}^{2}\right)}$ [36], $D_{t h}$ is thruster diameter, and $F_{t h}$ is thruster force that is determined from (16).

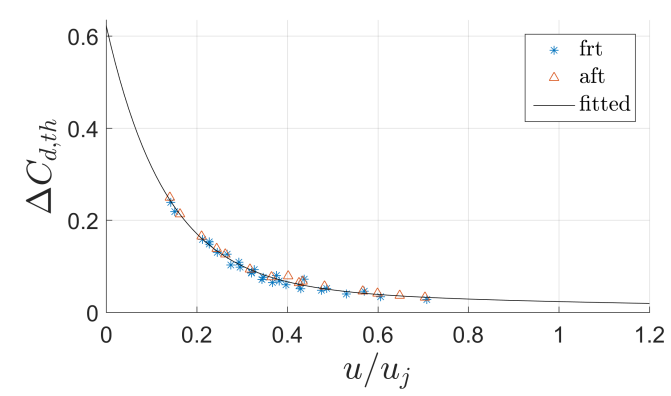

Figure 3: A change in volumetric drag coefficient due to an operation of front and aft thruster (adapted from [35]).

The drag for a thruster operation takes a form:

$$
\Delta X_{t h}=-\frac{1}{2} \rho \nabla^{2 / 3}|u| u \Delta C_{d, t h},
$$

where $\nabla$ denotes the volumetric displacement of the AUV.

This is incorporated into the model as a generalised force along the longitudinal axis of the vehicle: $\Delta \tau=\left[\Delta X_{t h, v f}+\Delta X_{t h, v a}, 0,0\right]^{T}$, where subscription $v f$ and $v a$ are used to refer to the vertical-front and vertical-aft thruster respectively.

\subsection{Actuator Modelling}

Considering the system that has $m$ degrees of freedom and involves $n$ components of forces and moments from actuators, the generalised force vector $(\tau)$ due to actuators may be characterised by a form $[26]$ :

$$
\tau=\boldsymbol{T} f
$$

where $f=\left[f_{1}, f_{2}, f_{3}, \ldots, f_{n}\right]^{T} \in \Re^{n}$ is a column vector representing the total forces and moments expressed in actuator local frame; $\boldsymbol{T}=$ $\left[t_{1}, t_{2}, t_{3}, \ldots, t_{m}\right] \in \Re^{m \times n}$ is a force configuration matrix that transforms $f$ into equivalent forces and moments acting on the origin of $b$-frame.

\subsubsection{Main Propeller Modelling:}

Standard propeller theory [37] suggests that it is possible to characterise a thrust due to a propeller by advance ratio, $J$, and thrust coefficient, $K_{T, \text { prop }}$. They are given by:

$$
\begin{aligned}
J & =\frac{u}{n_{\text {prop }} D_{\text {prop }}}\left(1-w_{t}\right), \\
K_{T, \text { prop }} & =K_{T_{0, \text { prop }}}\left(1-\left[\frac{J}{c_{\text {prop }, 1}}\right]^{c_{\text {prop }, 2}}\right),
\end{aligned}
$$

where $w_{t}$ denotes wake fraction estimated from [3]; $D_{\text {prop }}$ is propeller diameter; the propeller speed $\left(n_{\text {prop }}\right)$, measured in rev/s, in relation to the propeller demand is found from the experiment to be

$$
n_{\text {prop }}=-0.0055 \mathrm{u}_{\text {prop }}^{2}+0.4136 \mathrm{u}_{\text {prop }}-1.7895 .
$$

The relation between $K_{T, p r o p}$ and $J$ from (12) is illustrated in figure 4.

To this end, the thrust due to the main propeller is expressed as:

$$
F_{\text {prop }}=\rho n_{\text {prop }}^{2} D_{\text {prop }}^{4} K_{T, p r o p}(1-t)
$$

where $t$ is thrust deduction estimated from [3].

Components of the force configuration matrix and force vector for the main propeller are:

$$
t_{1}=\left[\begin{array}{l}
1 \\
0 \\
0
\end{array}\right], \quad f_{1}=F_{\text {prop }}
$$




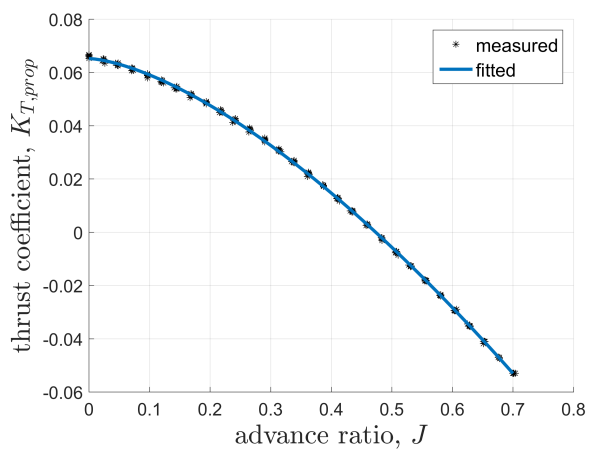

Figure 4: A $K_{T, p r o p}-J$ relation that is inferred from experiment data privided in [38].

\subsubsection{Thruster Modelling:}

A quasi-steady model is used for thruster modelling. Thrust deduction factors are included to model the thruster degradation due to forward and lateral motions of the AUV [39]. The force due to a thruster is given by

$$
\begin{aligned}
F_{t h}= & \underbrace{\rho\left|n_{t h}\right| n_{t h} D_{t h}^{4} K_{T, t h}}_{\text {static thrust }} \\
& \cdot \underbrace{e^{\left(-c_{t h, 1}(u)^{2}\right)} \cdot\left[1-c_{t h, 2}\left(\frac{v}{n_{t h} D_{t h}}\right)\right]}_{\text {deduction factors due to motions }}
\end{aligned}
$$

where $n_{t h}$ denotes a thruster speed, $D_{t h}$ denotes a thruster diameter, $K_{T, t h}$ is a thrust coefficient; constants for deduction factors were inferred from [35].

Components of the force configuration matrix and force vector for the thrusters are:

$$
t_{2}=\left[\begin{array}{cc}
0 & 0 \\
1 & 1 \\
L_{v f} & L_{v a}
\end{array}\right], \quad f_{2}=\left[\begin{array}{c}
F_{t h, v f} \\
F_{t h, v a}
\end{array}\right],
$$

where $L$ denotes the thruster arm length relative to a centre of rotation; subscriptions $v f$ and $v a$ refer to the vertical-front and vertical-aft thruster respectively (see also figure 7 ).

\subsubsection{Stern Plane Modelling:}

The lift, drag and moment due to stern planes are modelled using a hydrodynamic derivative according to [32]. They are given by:

$$
\begin{aligned}
X_{S} & =X_{|u| u \delta_{S} \delta_{S}}|u| u \delta_{S}^{2}, \\
Z_{S} & =Z_{|u| u \delta_{S}}|u| u \delta_{S}, \\
M_{S} & =M_{|u| u \delta_{S}}|u| u \delta_{S},
\end{aligned}
$$

where $\delta_{S}$ denotes stern plane deflection; the derivatives are deduced from results of wind tunnel testing on Delphin2 AUV [34].

Although the AUV rarely reverses in normal operations, it is possible that the forward speed becomes negative during acceleration/deceleration phases. Due to this reason, absolute signs are added to improve simulation stability when the speed is nearly zero. However, it is important to emphasise that stern plane dynamics is not forward/backwards symmetric, and this assumption is invalid for AUV operation at high reverse speeds.

Components of the force configuration matrix and force vector for the stern planes are:

$$
t_{3}=\left[\begin{array}{lll}
1 & 0 & 0 \\
0 & 1 & 0 \\
0 & 0 & 1
\end{array}\right], \quad f_{3}=\left[\begin{array}{c}
X_{S} \\
Z_{S} \\
M_{S}
\end{array}\right]
$$

\section{Control System Design}

In this section, two control strategies are developed for two operating conditions. The first is a hover-style control strategy that adjusts depth and pitch of the AUV by operating two vertical thrusters. The second is a flight-style control strategy that relies on stern planes operation for pitch angle control that, in turn, affects vehicle depth. Actuator weighting functions are used to provide a seamless transition between the two control strategies when operating over a wide range of speeds.

The controller gain values are identified empirically using a heuristic approach based on the Ziegler-Nichols method [40].

Note that, the PID control scheme in this work slightly differs from the convention: (1) a derivative term is placed in a feedback path instead of the forward path, (2) an actual state of the system is fed to the derivative term instead of the tracking error. (See figure 5.) Thus, the derivative terms are not affected by a sudden change in the required state, i.e., avoiding the setpoint kick phenomenon [40]. By doing so, the derivative gains must have a negative value; hence it is commonly referred to as PI-D scheme. 


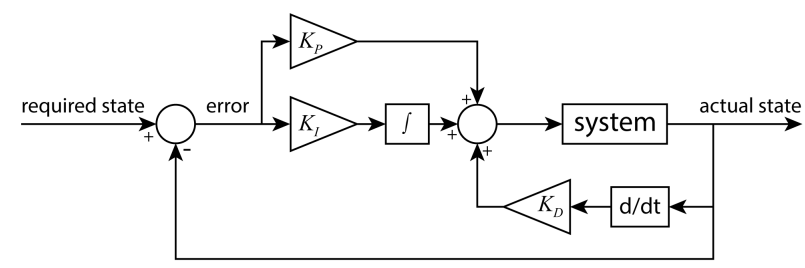

Figure 5: PI-D scheme.

\subsection{Hover-Style Control Strategy}

A control strategy for hover-style operation is used to continuously operate the two vertical thrusters to counter a net positive buoyancy, see figure 6 . It is also important that the forces from the two thrusters are balanced. Otherwise, the AUV will end up pitching and the thrust components will cause the AUV to move forward/backwards.

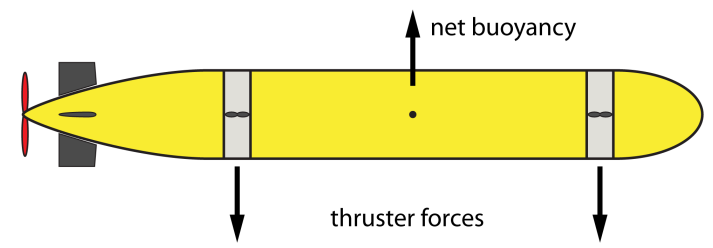

Figure 6: Hover-style force diagram.

The AUV is considered as a rigid body that needs to be pushed down by a generalised force $Z$, see figure 7 . Based on a depth error signal $(\tilde{z})$, a generalised force control law is

$$
Z=K_{P, Z} \tilde{z}+K_{I, Z} \int \tilde{z} \mathrm{~d} t+K_{D, Z} \dot{z} .
$$

If a virtual centre of rotation ( $c r$ ) is known, the total force $Z$ can be allocated between the two thrusters so that the pitch error signal $(\tilde{\theta})$ is driven to zero. To this end, the $c r$ shift control law is

$$
\Delta c r=K_{P, c r} \tilde{\theta}+K_{I, c r} \int \tilde{\theta} \mathrm{d} t+K_{D, c r} \dot{\theta} .
$$

From figure 7, given an initial guess for a centre of rotation $\left(c r_{0}\right)$, the virtual centre of rotation is computed: $c r=c r_{0}+\Delta c r$. Arm lengths for the front $\left(L_{v f}\right)$ and aft $\left(L_{v a}\right)$ vertical thrusters are determined accordingly and are used for a force

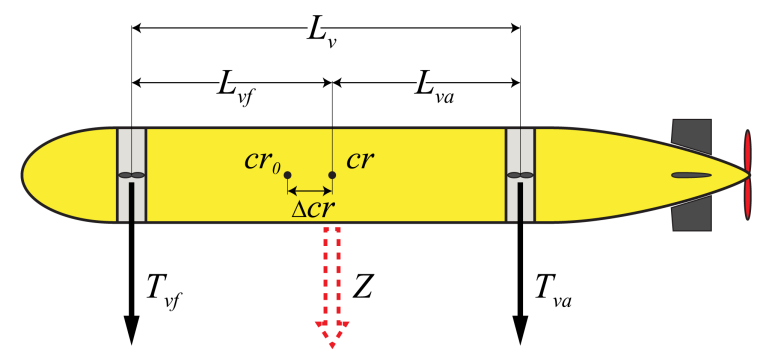

Figure 7: Parameters used for a generalised force allocation.

allocation:

$$
\left[\begin{array}{l}
T_{v f} \\
T_{v a}
\end{array}\right]=\left[\begin{array}{l}
L_{v f} / L_{v} \\
L_{v a} / L_{v}
\end{array}\right] Z .
$$

Finally, forces are then converted to a thruster demand:

$$
\mathrm{u}_{t h}=\operatorname{sign}(T) \sqrt{\frac{|T|}{\rho D_{t h}^{4} K_{T, t h}}} .
$$

Note that the thruster demand $\left(\mathrm{u}_{t h}\right)$ is equivalent to the thruster speed $\left(n_{t h}\right)$, and $(25)$ is in fact an inverse of (16) where the deduction factors are neglected.

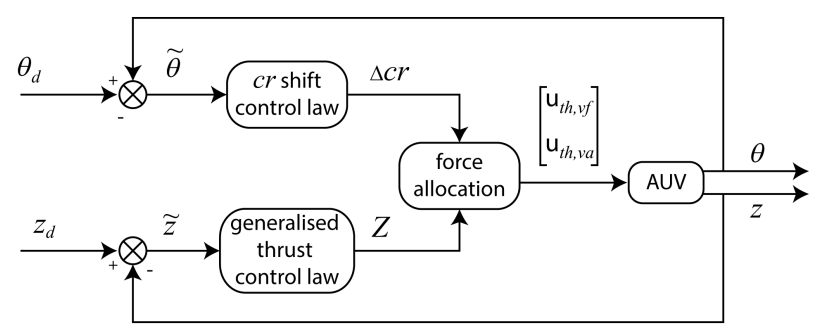

Figure 8: A hover-style control block diagram.

The hover-style control strategy is illustrated as a control block diagram in figure 8 .

\subsubsection{Anti-Windup Scheme:}

It is found that an integrator for the generalised thrust control law in (22) may become unnecessary large when dealing with a significant change in a depth demand. This phenomenon is known as integral windup. This has a severe impact on the control performance because the system needs to produce an opposite sign of error signal, i.e., overshoot, just to unwind the integrator. 
A conditional integration technique is used to reduce the effect of the integral windup phenomenon. The integrator will be frozen if $|\tilde{z}|>$ $\tilde{z}_{\text {sat }}$ or $|\dot{z}|>\dot{z}_{\text {sat }}$. That is, do not update the integrator if the depth error is significantly large or the depth is already changing quickly enough. In either case, a further effort from the integrator is not yet required for steady-state error compensation.

\subsection{Flight-Style Control Strategy}

The hover-style control strategy described in the previous section cannot be used effectively at high forward speeds. This is because thruster performance decreases when forward speed increases [39]; consequently, the power consumption becomes unsustainable. Also, the AUV hull behaves like a large, low aspect ratio aerofoil, generating a hydrodynamic force that dominates the forces produced by the thrusters.

In the high-speed regime, using stern planes is, therefore, a better strategy since this is more effective and requires less energy. The AUV pitch is adjusted by using the stern planes in order to balance the hydrodynamic force with the net buoyancy. This is illustrated in figure 9 .

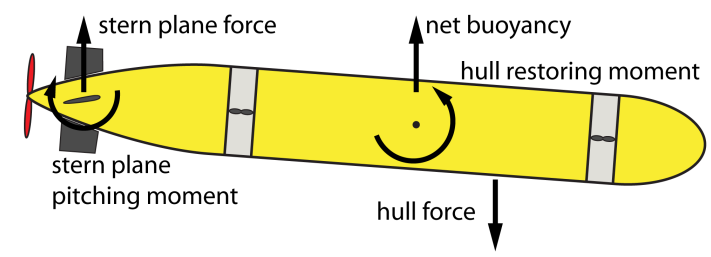

Figure 9: Flight-style force diagram.

To this end, a pitch demand is determined from a pitch bias control law:

$$
\theta_{d}=K_{P, \theta} \tilde{z}+K_{I, \theta} \int \tilde{z} \mathrm{~d} t+K_{D, \theta} \dot{z} .
$$

Then, the pitch error signal is used to determine a required stern plane deflection, $\delta_{S}$, as follows:

$$
\delta_{S}=K_{P, S} \tilde{\theta}+K_{I, S} \int \tilde{\theta} \mathrm{d} t+K_{D, S} \dot{\theta}
$$

These steps are illustrated as a control block diagram in figure 10.

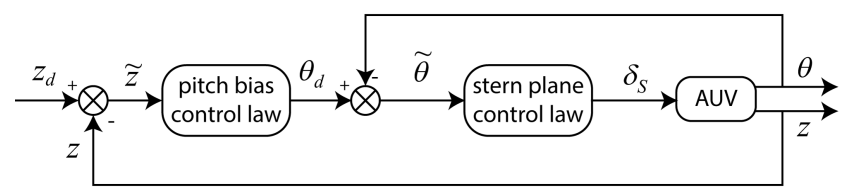

Figure 10: A flight-style control block diagram.

\subsection{Transition between two Control Strategies}

Control strategies from section 4.1 and 4.2 are unified into one control system that works over an entire range of operating speeds.

This is done by applying actuator weighting functions as a gain to manage the contribution of each set of actuators according to the forward speed (figure 11). These functions are:

$$
\begin{aligned}
& w_{t h}=1-\frac{1}{2}\left(\tanh \left(\frac{u-u_{t h}^{*}}{\sigma_{t h}^{*}}\right)+1\right), \\
& w_{S}=\frac{1}{2}\left(\tanh \left(\frac{u-u_{S}^{*}}{\sigma_{S}^{*}}\right)+1\right)
\end{aligned}
$$

where $u_{t h}^{*}$ and $u_{S}^{*}$ are a mid-transition speed and $\sigma_{t h}^{*}$ and $\sigma_{S}^{*}$ are a width of transition zone [22].

As speed increases, thruster weight, $w_{t h}$, decreases from 1 to 0 ; therefore, the use of thrusters is entirely removed at the high-speed regime. By contrast, the reliance on stern planes gradually increases with speed.

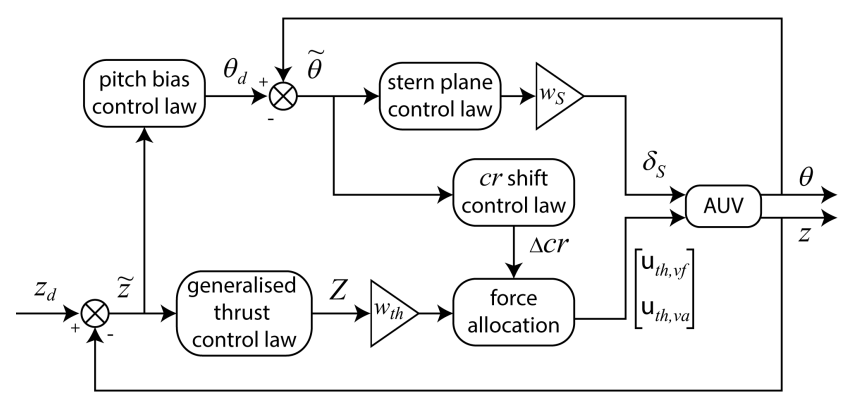

Figure 11: A unified diving control system: flight-style and hover-style strategies are combined.

A critical speed [3, p.169], below which stern planes become ineffective, is experimentally found to be approximately $0.7 \mathrm{~m} / \mathrm{s}$. To ensure a sufficient control authority, the weighting functions are designed to stop using the thrusters after this critical speed and start using the stern planes before this speed. This results in weighting func- 
tions for thruster and stern planes that are overlapping over the intermediate speed regime, see figure 12 .

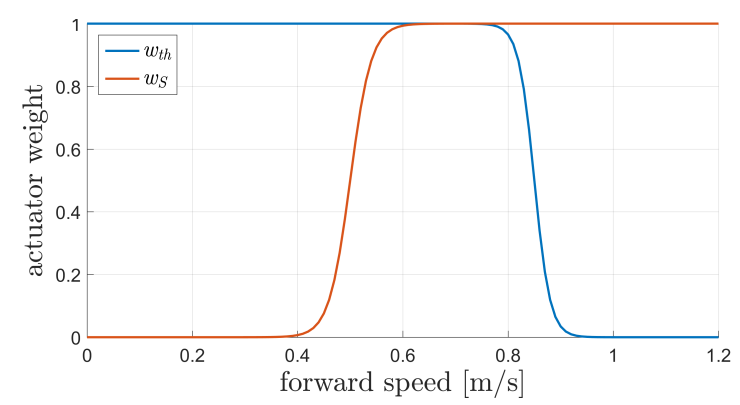

Figure 12: Actuator weighting functions: $u_{t h}^{*}=0.85$, $\sigma_{t h}^{*}=0.03, u_{S}^{*}=0.5$ and $\sigma_{S}^{*}=0.04$.

\section{Stability and Convergence Analysis}

A Lyapunov-based approach is used to analyse the system stability. The dynamics model, (1), is simplified by excluding the surge dynamics. Also, it is assumed that the pitch angle, $\theta$, is negligibly small; hence, $\boldsymbol{J}(\eta)$ becomes an identity matrix. This gives a following form of a heave-pitch dynamics model:

$$
\begin{gathered}
\boldsymbol{M} \cdot \dot{\nu}+\boldsymbol{C}(\nu) \cdot \nu+\boldsymbol{D}(\nu) \cdot \nu=\tau, \\
\dot{\eta}=\nu,
\end{gathered}
$$

where $\nu=[w, q]^{T}, \eta=[z, \theta]^{T}, \tau=[Z, M]^{T}$ and the error tracking vector is $\tilde{\eta}=\eta_{d}-\eta=[\tilde{z}, \tilde{\theta}]^{T}$. The hydrostatic force vector, $g(\eta)$, is assumed to be cancelled out by the integral terms in the control system; hence, they are not considered in the stability analysis.

The control reference in the stability analysis is assumed a constant, hence $\dot{\eta}_{d}=0$. This leads to: $\dot{\tilde{\eta}}=-\dot{\eta}=-\nu$.

Note that, for the proposed hover-style control strategy, $Z$ and $M$ are defined by (22) and (23), respectively. On the other hand, for the proposed flight-style control strategy, $Z$ and $M$ are defined by (26) and (27), respectively.

A Lyapunov function candidate is designed based on kinetic and potential energy of the system [26, p.376]:

$$
V=\frac{1}{2} \nu^{T} \boldsymbol{M} \nu+\frac{1}{2} \tilde{\eta}^{T} \boldsymbol{K}_{P} \tilde{\eta},
$$

where $\boldsymbol{K}_{P}>0$ is a constant, proportional-gain, diagonal matrix. A time differentiation of the Lyapunov function candidate is:

$$
\begin{aligned}
\dot{V} & =\nu^{T} \boldsymbol{M} \dot{\nu}+\tilde{\eta}^{T} \boldsymbol{K}_{P} \dot{\tilde{\eta}} \\
& =\nu^{T}\left[\boldsymbol{M} \dot{\nu}-\boldsymbol{K}_{P} \tilde{\eta}\right] .
\end{aligned}
$$

By substituting (30) into (33), this yields:

$$
\dot{V}=\nu^{T}\left[\tau-\boldsymbol{C}(\nu) \nu-\boldsymbol{D} \nu-\boldsymbol{K}_{P} \tilde{\eta}\right]
$$

The generalised control force vector is chosen, according to the PI-D scheme, to be:

$$
\tau=\boldsymbol{K}_{P} \tilde{\eta}+\boldsymbol{K}_{D} \dot{\eta}
$$

where $\boldsymbol{K}_{D}$ is a constant, derivative-gain, diagonal matrix. (This complies with the proposed control structure for both hover and flight styles.) Substitute this generalised force vector into (34) to give:

$$
\dot{V}=\nu^{T}\left[\boldsymbol{K}_{P} \tilde{\eta}+\boldsymbol{K}_{D} \dot{\eta}-\boldsymbol{C}(\nu) \nu-\boldsymbol{D} \nu-\boldsymbol{K}_{P} \tilde{\eta}\right] .
$$

Since $\nu^{T} \boldsymbol{C}(\nu) \nu=0$ for all $\nu$ and $\dot{\eta}=\nu$, then the above equation becomes:

$$
\dot{V}=-\nu^{T}\left[-\boldsymbol{K}_{D}+\boldsymbol{D}\right] \nu .
$$

It is known that $\nu^{T} \boldsymbol{D} \nu>0$. With $\boldsymbol{K}_{D}<0$, the Lyapunov stability criterion, $\dot{V}<0$, is satisfied, ensuring a global asymptotically stable.

Furthermore, at an equilibrium point $(\dot{V}=0)$, (33) becomes:

$$
\dot{\nu}=\boldsymbol{M}^{-1} \boldsymbol{K}_{P} \tilde{\eta}
$$

This suggests, the system could only converge to an equilibrium point where $\tilde{\eta}=[0,0]^{T}$, and will not get stuck at any other equilibrium points.

\section{Simulation Parameters}

All simulations were performed in Matlab Simulink with parameters provided in Appendix B. Initial conditions were $\nu=[0,0,0]^{T}$ and $\eta=$ $[0,0,0]^{T}$, i.e., the AUV is at rest on the water surface.

The solver was Runge-Kutta $4^{\text {th }}$ order. Sample rates were fixed by the IMU at $20 \mathrm{~Hz}$ for the dynamics modelling section and $5 \mathrm{~Hz}$ for the control section. These comply with actual sample rates used on Delphin2 AUV. 


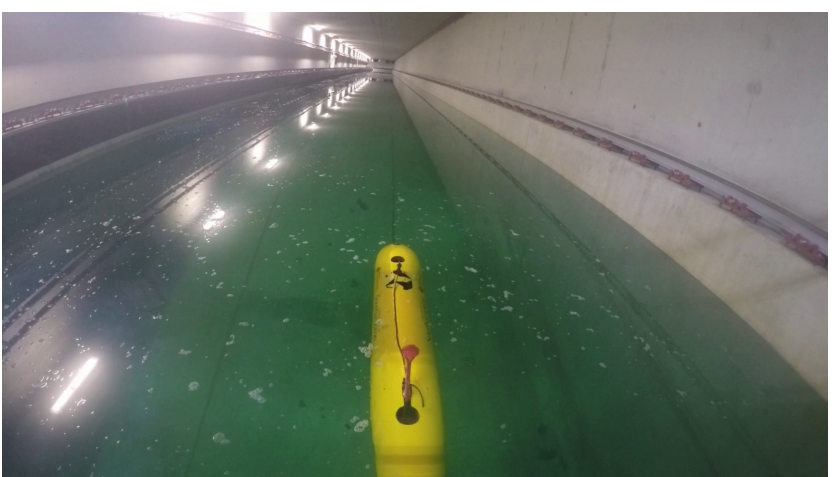

(a) Alignment setup

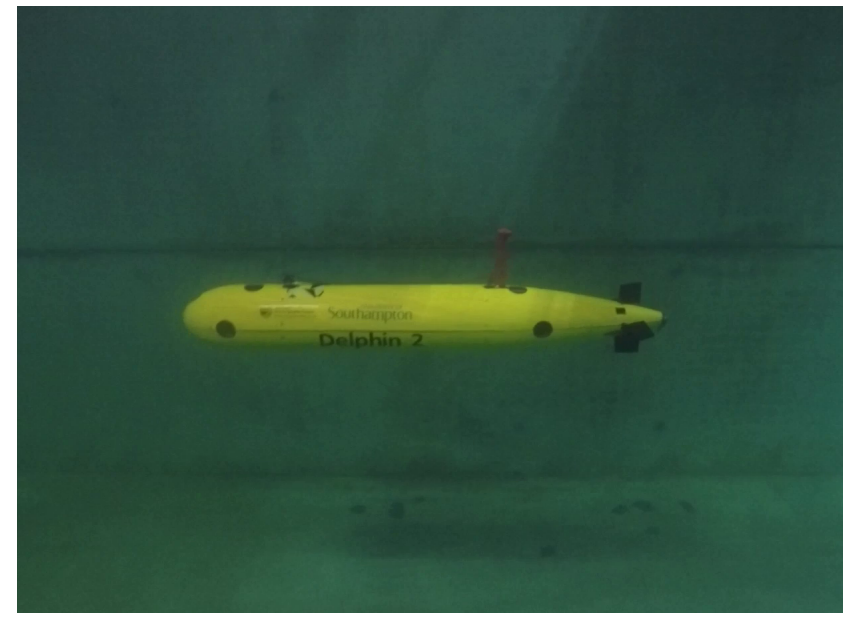

(b) Delphin2 AUV is hovering at constant depth

Figure 13: Experiment performed in the Boldrewood towing tank of dimensions $138 \mathrm{~m}$ long $\mathrm{x} 6 \mathrm{~m}$ wide $\mathrm{x} 3.5 \mathrm{~m}$ deep.

\section{Experiment Setup}

The proposed control system was implemented on the Delphin2 AUV at $5 \mathrm{~Hz}$ so that it can synchronise to the sensor and actuator interfacing nodes.

All tests were performed in the freshwater Boldrewood towing tank, see figure 13. Each test was started when the AUV was at rest on the water surface, positioned in the centre of the tank width, and aligned with the tank length.

A heading controller was active to maintain a constant heading. A reference heading was obtained from the Xsens $4^{\text {th }}$ generation MTi-30 IMU. Since the tank features an extreme magnetic disturbance, the utilised heading is purely based on gyroscope and accelerometer responses.

Propeller demands used in these tests were $\mathrm{u}_{\text {prop }}=\{0,10,16,22\}$ — these numbers are set- points that the motor control board requires when driving the motor. They approximately correspond to forward speeds of $u=\{0,0.26,0.6,1.0\}$ $\mathrm{m} / \mathrm{s}$ respectively and are referred to in the result section as zero-, low-, mid- and high-speed cases.

When the AUV is at the surface, the top sides of the vertical thrusters are not sufficiently submerged for efficient operation. Due to this, the integrator in the generalised thrust control law was preloaded by 1.2 times of the integrator value in a steady-state. As a result, the AUV was able to dive as soon as the mission starts. Previous experiments have shown that a depth of approximately $0.1 \mathrm{~m}$ is sufficient for the thrusters to function effectively [41].

\section{Results and Discussion}

There are two scenarios used for testing system stability: the first is zero-speed operation, aiming to demonstrate performance for the hoverstyle control strategy; the second is operation at a constant forward speed with initial and final operation at zero speed, aiming to demonstrate the performance when the vehicle is accelerating from at rest to a constant speed and vice versa.

Additional studies on power consumption and sensitivities to disturbances are also presented.

\subsection{Hover Style Operation at Zero-speed}

In each run, the AUV started from the surface and is pre-programmed with three consecutive depth demands of 1,2 and $1 \mathrm{~m}$. Simulation and experimental results are presented in figure 14.

The experimental and simulation results illustrate the same qualitative behaviours. However, the experiments exhibit a reduced overshoot, and the oscillations are more heavily damped. With the conventional integrator, the vehicle takes less than $30 \mathrm{sec}$ to settle and eventually converges to the desired depth with no steady-state error (figure 14a). As the depth demand changes, thruster demands are generated to accelerate the AUV toward the desired depth. These quickly converge to the level that is needed to counter the net positive buoyancy. 

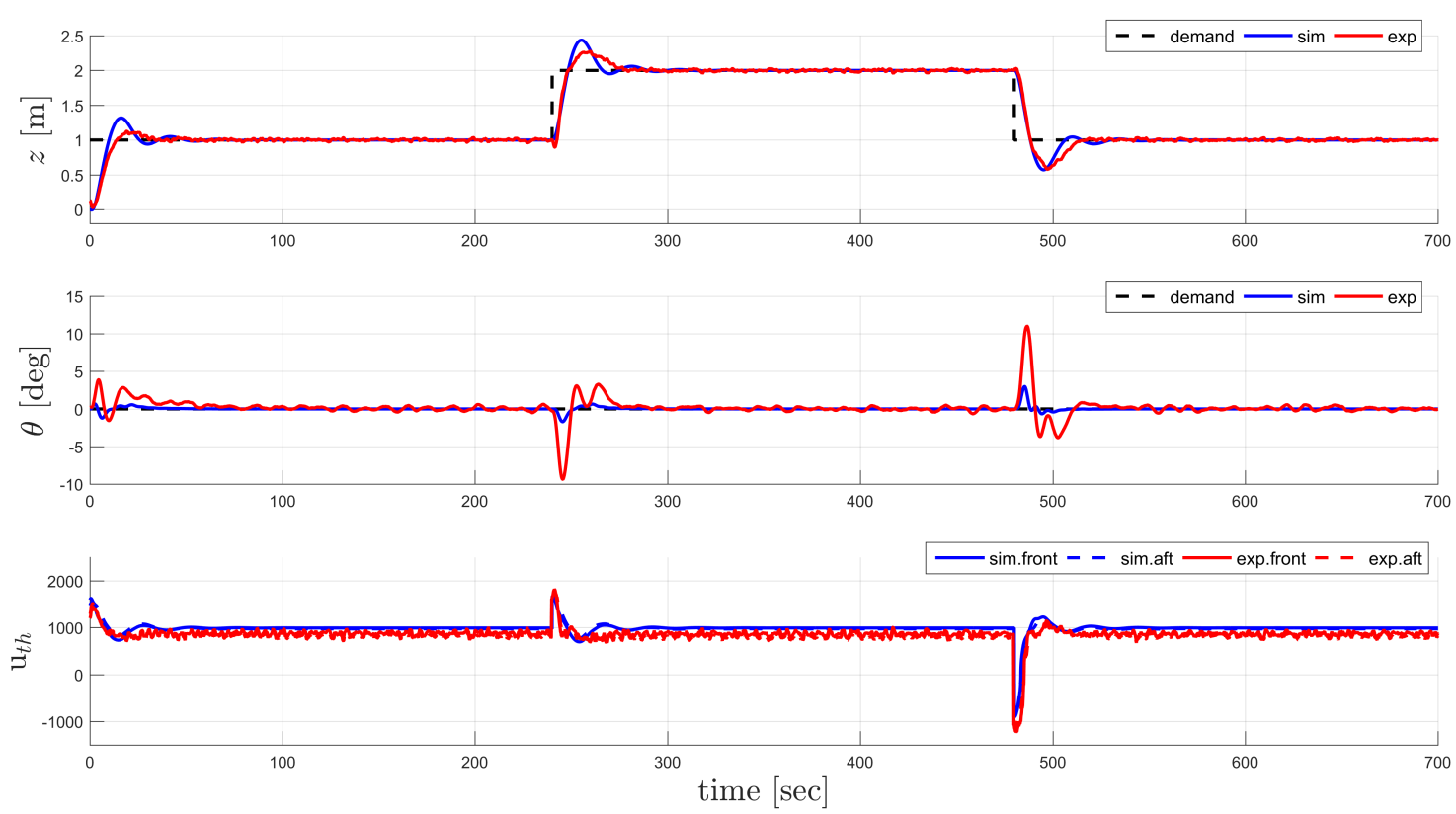

(a) without anti-windup scheme
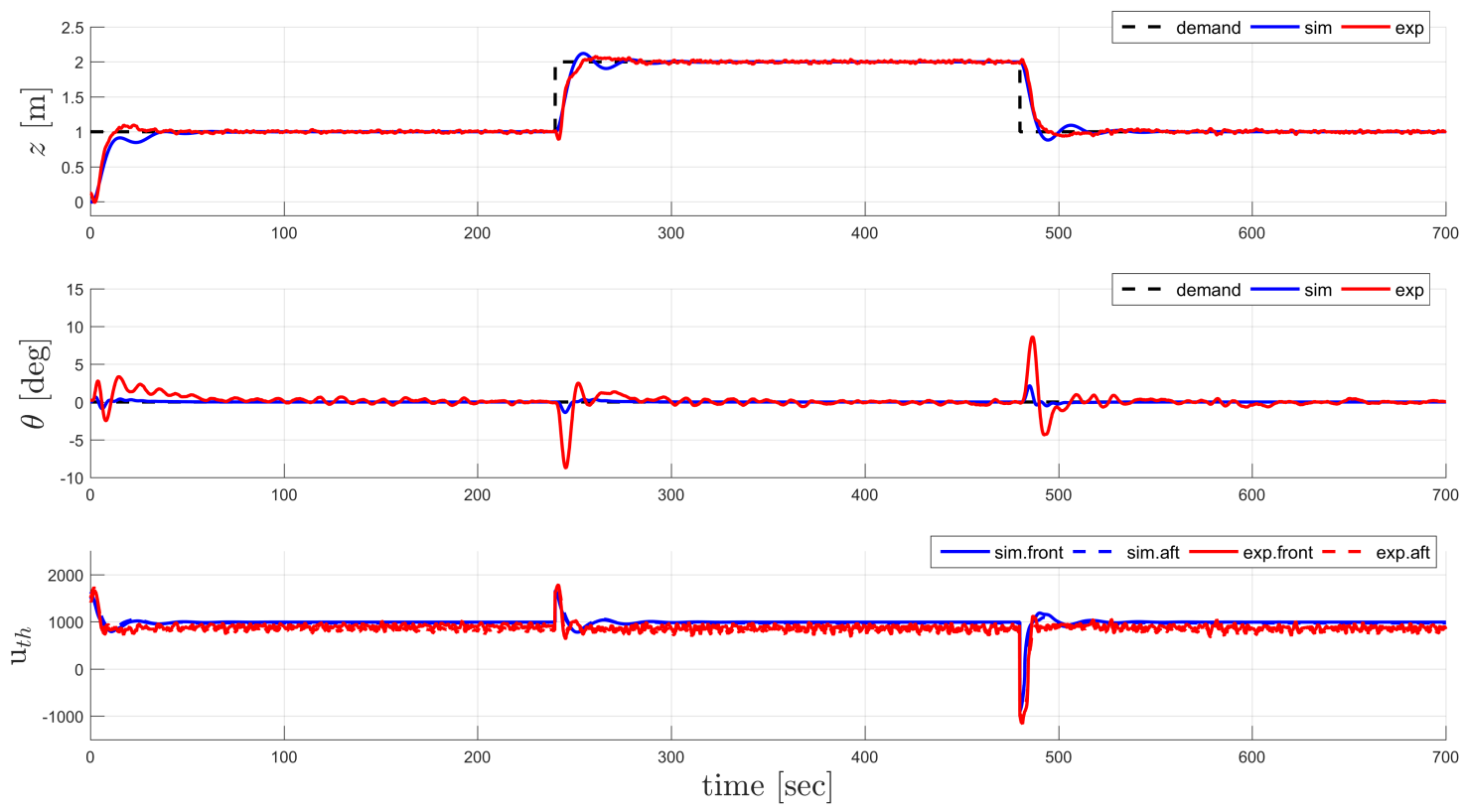

(b) with anti-windup scheme

Figure 14: Hover-style operation at zero-speed.

There is, however, a considerable overshoot in depth (approximately $0.5 \mathrm{~m}$ ) which occurs when the depth demand changes. This is due to the conventional integrator continuously accumulating the error signal. Hence, the integrator momentarily becomes unnecessary huge, i.e., windup, when dealing with a significant change in step demand or when a transient is long. The system needed to overshoot just to produce an opposite sign of error signal so that the integrator is unwound.

To improve the transient performance, the antiwindup scheme, described in section 4.1.1, was imposed to freeze the integrator when a steadystate error compensation is not yet required. As shown in figure 14b, there is a significant improvement in overshoot which reduces to about $0.2 \mathrm{~m}$, and the settling time is reduced to less than 15 
sec. Also, the integrator with anti-windup scheme is more stable during the transient, see figure 15 .

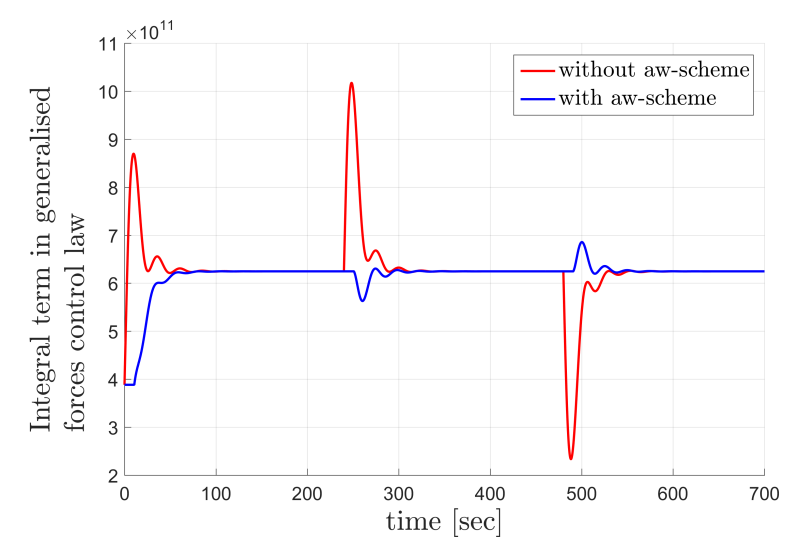

Figure 15: A comparison on the integrators from simulation results, tracking three consecutive depth demands of 1,2 and $1 \mathrm{~m}$.

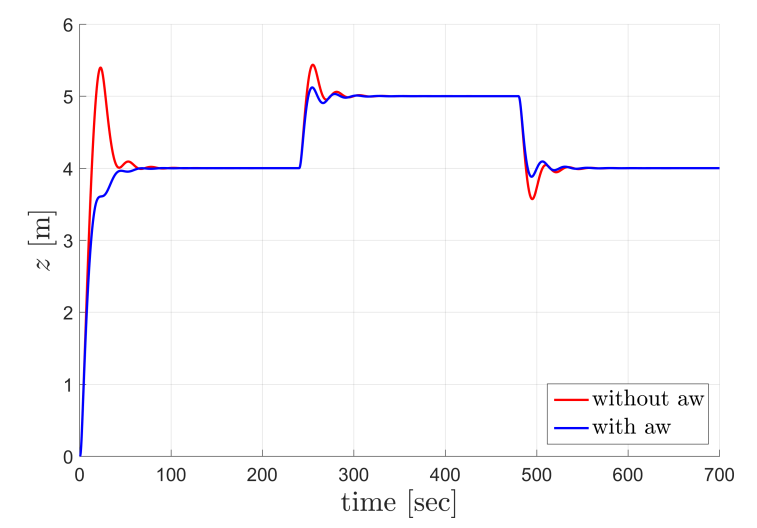

Figure 16: Simulation results for a comparison of depth tracking performance with different sizes of changes in step depth demands.

It is also important to note that, with the conventional integrator, the magnitude of the overshoot grows in proportion to the size of a step change in the depth demand. The overshoot may be as high as $1 \mathrm{~m}$ for $4 \mathrm{~m}$ depth change which is considered unacceptably large, see figure 16 . However, this issue can be alleviated when the anti-windup scheme is imposed.

Considering the pitch tracking error in figure 14, the AUV is pitching towards the desired depth when initially adjusting the depth. This is due to the presence of the stern planes at the far aft that influences pitch dynamics when undergoing heave motions. The degree of pitch variation from the experiment is more significant, and may be as high as $\pm 10 \mathrm{deg}$. This implies that the value of $M_{|q| q}$ is underpredicted. However, the ratio of forces between front and rear thruster is adapting to quickly dampen the pitch dynamics.

\subsection{Operation at Depth with a Speed Transition}

Each run starts when the AUV is at rest on the water surface. The AUV is demanded to dive vertically to $1 \mathrm{~m}$ depth at a zero propeller demand. It is then programmed to maintain depth while executing a constant propeller demand for a fixed period of time. Following this, the propeller stops and the AUV needs to maintain depth at a zero propeller demand for $30 \mathrm{sec}$ before the run is finished, and the vehicle rises to the surface under its positive buoyancy.

The simulation and experimental results from flight-style operation are presented in figure B.22. These show that the proposed control system yields a good depth tracking performance over an entire speed range. Although there is a slight deviation in depth during the acceleration/deceleration phase, the fluctuation is bounded within $\pm 0.2 \mathrm{~m}$ (considered practically acceptable) and quickly decays within 20 sec.

In the low-speed case, the AUV is able to maintain depth with no steady-state depth error by using only the thrusters. However, since $\Delta c r$ is bounded, the pitch moment generated by the thrusters is also limited, and this results in a steady-state error in pitch.

As forward speed increases, the controller uses the thrusters less and relies on the stern planes more. When operating at mid-speed and highspeed, the vehicle needs to pitch nose down by -5 deg approximately to balance the net buoyancy force.

When comparing to the results obtained with the MPC approach implemented on the same vehicle under the same ballast condition, there is a significant improvement in the control performance. The evidence in [31, p.186] shows that, when the MPC technique is used, the AUV pitches down by -20 and -10 deg in low- and midspeed operation respectively; such a large angle of attack leads to a significant decrease in the 

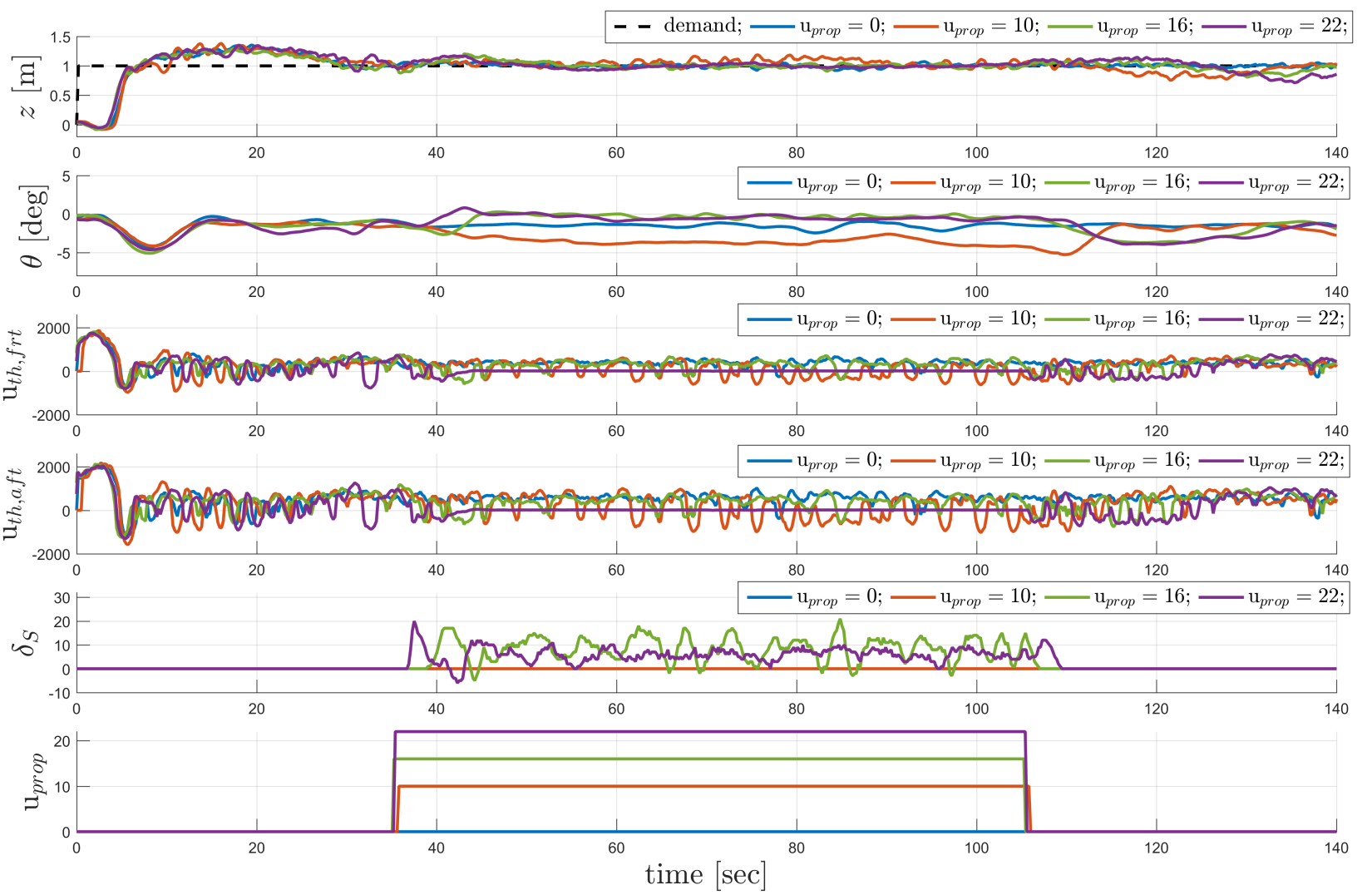

Figure 17: Experimental results show control performance for the AUV with $+1 \mathrm{~N}$ net buoyancy.

vehicle efficiency. Also, the AUV's depth deviates by approximately $0.5 \mathrm{~m}$ when accelerating/decelerating, and it requires up to $60 \mathrm{sec}$ to recover. Now, with the proposed control system, the AUV's depth only deviates by $\pm 0.2 \mathrm{~m}$ and quickly decays within $20 \mathrm{sec}$. Also, during lowand mid-speed operation, the AUV only pitches down by -5 deg at most.

\subsection{Influence of Positive Buoyancy}

The actuator demands during steady operation are highly dependent on the magnitude of the net buoyancy. The impact on controller performance is considered in this subsection. The procedure for testing with a speed transition, described in the previous subsection, is repeated for Delphin2 ballasted to be almost neutrally buoyant (the residual buoyancy is measured to be $+1 \mathrm{~N}$ ); this buoyancy condition remains unchanged for every case in this scenario.

Experimental results in figure 17 show that the almost neutrally buoyant AUV could steadily maintain a constant depth for the entire range of propeller setpoints as well as during the acceleration/deceleration phases. Thruster setpoints mostly fluctuate around the zero value instead of holding at a constant demand like they do for the typical buoyancy $(+6 \mathrm{~N})$ case. In addition, it is described in section 7 that the integral term is preloaded so that the AUV with the typical buoyancy can quickly dive. However, this preloading technique only delivers the best performance for a certain buoyancy condition. For the neutrally buoyant cases, the pre-loading causes $0.3 \mathrm{~m}$ overshoot in depth before the integral term unwinds and the AUV gets back to the desired depth within 35 sec. 


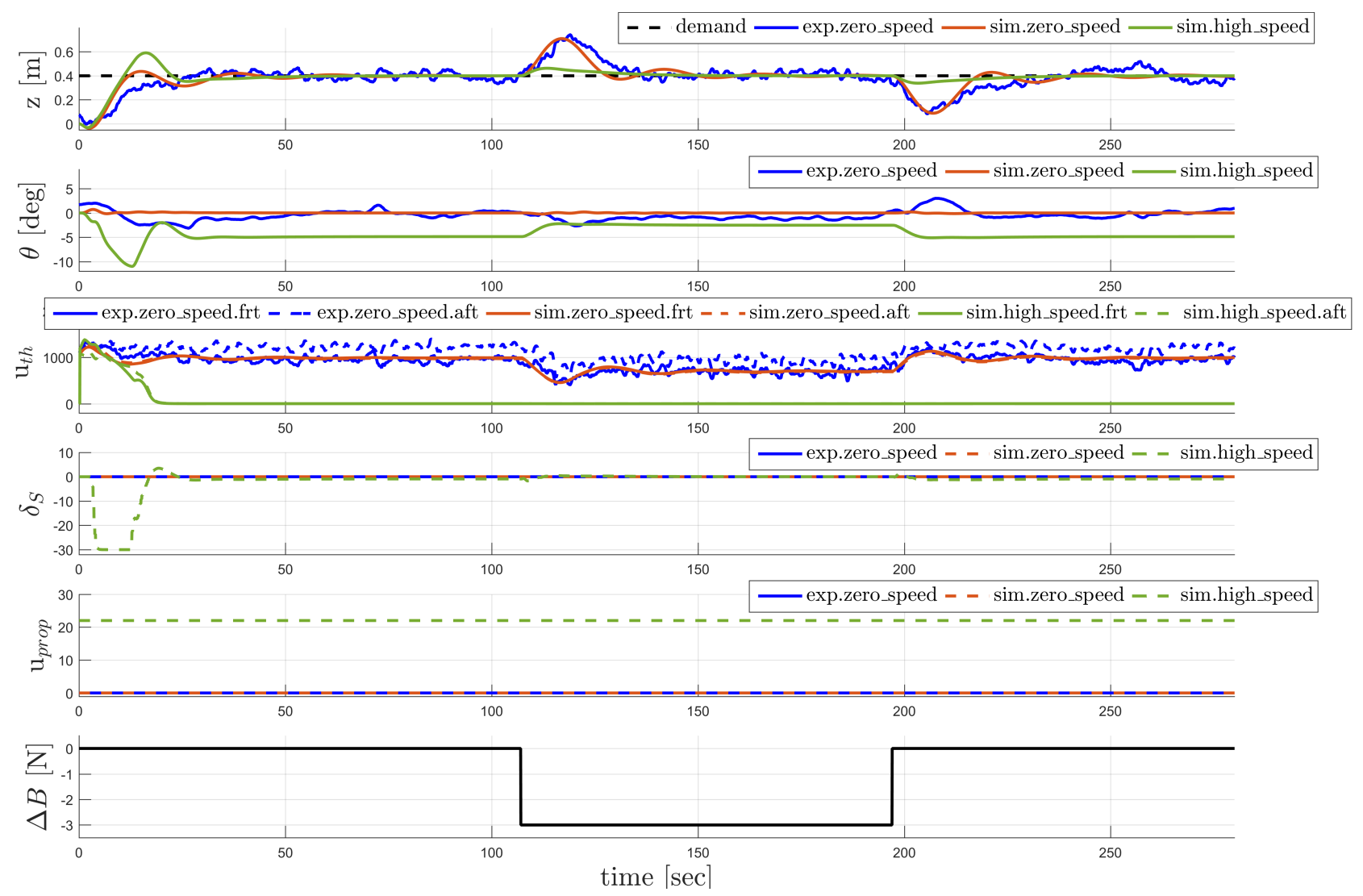

Figure 18: Results show control performance when the system is subjected to a variation in the net buoyancy.

\subsection{Sensitivity to Large Disturbance}

An effect of a sudden change in the net buoyancy on the control performance is examined by simulation for both zero- and high-speed cases: the AUV is demanded to maintain constant depth at constant propeller demand, then an extra weight is added and, later on, removed.

A $3 \mathrm{~N}$ variation in buoyancy is considered. Such a change could be observed in operation in areas of varying salinity. An experiment for the same scenario was carried out on the AUV for only zero speed because the ballast needed to be manually removed during the experiment.

Figure 18 shows that when a $3 \mathrm{~N}$ change in the net buoyancy suddenly occurs, the AUV depth tracking performance momentarily degrades, and the AUV depth deviates from the depth setpoint by $0.3 \mathrm{~m}$. It then recovers within $20 \mathrm{sec}$ by winding/unwinding the integral terms in the control laws. In the zero-speed case, the system adapts by adjusting the thruster setpoints so they match to the new net buoyancy. On the other hand, in the high-speed case, the system adapts by adjusting the AUV pitch through the use of stern planes.

\subsection{Energy Consumption}

The power consumption, $P_{t o t}$, of a neutrally buoyant flight style AUV can be assumed to be split between hotel load, $P_{H}$, which is invariant to forward speed, and propulsion power, $P_{P}$, which is related to propulsion speed raised to the power 3 [42, 43]. For a positively buoyant over-actuated vehicle, additional power is required to maintain depth, $P_{B}$ :

$$
P_{\text {tot }}=P_{H}+P_{P}+P_{B}
$$

Power consumption profiles, for the Delphin2 AUV operating at constant depth with a typical $(+6 \mathrm{~N})$ and almost neutral $(+1 \mathrm{~N})$ buoyancy, are presented in figure B.23, and tables 1 and 2; they are compared to predicted power consumption for an ideal neutrally buoyant AUV with the same propulsion power requirements and hotel load. 
Table 1: Average power required when operating at constant depth at different speeds ( $+6 \mathrm{~N}$ buoyant).

\begin{tabular}{|c|c|c|c|c|c|c|c|c|}
\hline \multirow[b]{2}{*}{$\mathrm{u}_{\text {prop }}$} & \multirow{2}{*}{$\begin{array}{c}u \\
{[\mathrm{~m} / \mathrm{s}]}\end{array}$} & \multicolumn{6}{|c|}{ power [W] } & \multirow{2}{*}{$\begin{array}{c}\text { COT } \\
{[\mathrm{kJ} / \mathrm{kg} / \mathrm{km}]}\end{array}$} \\
\hline & & hotel & th-frt & th-aft & fins & prop & total & \\
\hline 0 & 0.00 & 30 & 10.73 & 11.11 & 0.21 & 0.00 & 52.05 & - \\
\hline 10 & 0.26 & 30 & 12.62 & 13.17 & 0.16 & 3.12 & 59.06 & 2.86 \\
\hline 16 & 0.60 & 30 & 11.98 & 13.05 & 1.00 & 11.77 & 67.80 & 1.42 \\
\hline 22 & 1.00 & 30 & 1.42 & 5.40 & 0.89 & 22.19 & 59.90 & 0.75 \\
\hline
\end{tabular}

Table 2: Average power required when operating at constant depth at different speeds $(+1 \mathrm{~N}$ buoyant $)$

\begin{tabular}{|c|c|c|c|c|c|c|c|c|}
\hline \multirow[b]{2}{*}{$\mathrm{u}_{\text {prop }}$} & \multirow{2}{*}{$\begin{array}{c}u \\
{[\mathrm{~m} / \mathrm{s}]}\end{array}$} & \multicolumn{6}{|c|}{ power [W] } & \multirow{2}{*}{$\begin{array}{c}\text { COT } \\
{[\mathrm{kJ} / \mathrm{kg} / \mathrm{km}]}\end{array}$} \\
\hline & & hotel & th-frt & th-aft & fins & prop & total & \\
\hline 0 & 0.00 & 30 & 2.22 & 3.59 & 0.58 & 0.00 & 36.39 & - \\
\hline 10 & 0.32 & 30 & 3.31 & 2.79 & 0.58 & 2.74 & 39.42 & 1.86 \\
\hline 16 & 0.68 & 30 & 2.24 & 2.12 & 1.25 & 11.79 & 47.40 & 1.04 \\
\hline 22 & 1.01 & 30 & 0.12 & 0.55 & 1.11 & 21.29 & 53.07 & 0.77 \\
\hline
\end{tabular}

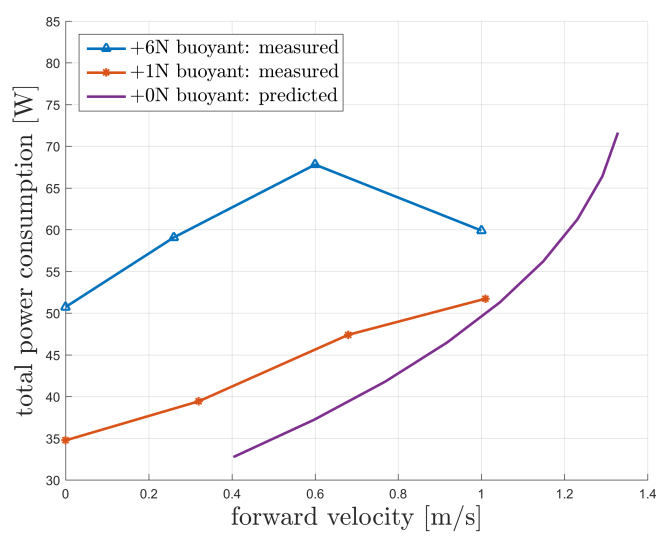

Figure 19: Power consumption at different speeds.

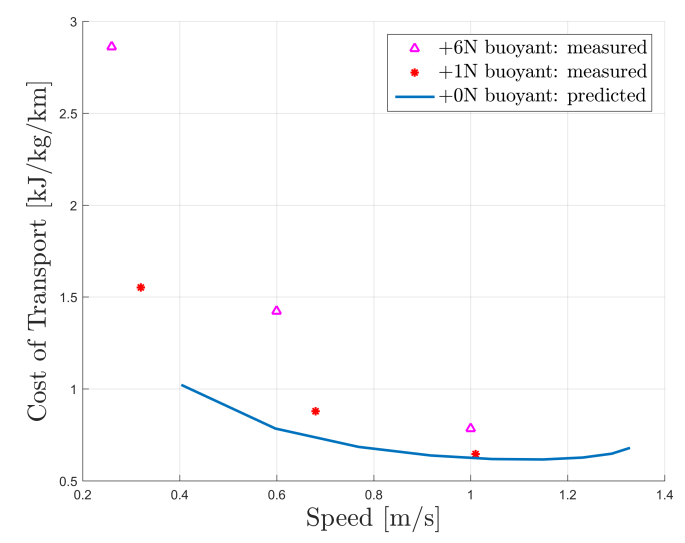

Figure 20: Cost of transport (COT) at different speeds.

The results presented here indicate that, for the typical buoyancy cases, $P_{B}$ dominates at slow speeds due to the comparative inefficiency of thrusters in generating stationary thrust. A sudden decrease in total power consumption occurs at high speeds when the thruster is switched off, and only the stern planes are used to maintain depth, see figure 19. This decrease in power consumption is also directly linked to the selected weighting function. The mid-transition speed in the thruster weighting function could be modified to lower the energy spent on thrusters earlier, e.g., starts from mid-speed onwards. However, a greater pitch down angle, up to -20deg, is required as a trade-off [31], and, below a critical speed, the AUV will be unable to maintain control authority using the stern planes alone [12].

The total power requirement may be analysed as a cost of transport (COT), which is a normalised measure of the energy required to transport the mass of the vehicle over a unit distance at a speed $u$. The general formulation of the cost of transport (COT) for an individual is given by

$$
C O T=\frac{P_{H}+P_{P}+P_{B}}{m u} .
$$

Figure 20 concludes that it is more energy efficient for the AUV to perform the flight-style operation at high speeds, with an optimal forward speed of approximately $1.1 \mathrm{~m} / \mathrm{s}$. As the AUV becomes more neutrally buoyant, COT tends to converge to the predicted COT for the ideal AUV.

This suggests that, for hover-style operation, a large amount of energy could be saved by employing a buoyancy engine to actively adjust the net buoyancy to as close to zero as possible; hence, fewer efforts are required for holding the AUV at depth. The primary concern is that this solution requires significant modifications to the present AUV. It also needs to mention that the typical buoyancy engine response is slow, and the vertical thrusters are still needed for faster actions. 


\section{Conclusions}

This paper presents a robust PI-D based controller for operation of an over-actuated, hover-capable AUV. By utilising an appropriate weighting function, the appropriate allocations of control forces are shared across actuators. Stability and convergence are proven using a Lyapunov-based approach. The controller is shown to provide good performance both in simulations and experiments. Typical depth overshoots/variations of less than $0.2 \mathrm{~m}$ are observed when charging depth or speed. Such small deviations are acceptable operationally and are consistent with or better than results using more computationally expensive MPC scheme for the same vehicle.

The impact of net positive buoyancy is considered, and its impact on control performance is explained. In low speeds, as the positive buoyancy decrease, less effort from thrusters are required to maintain a constant depth. On the other hand, the impact is subtle for high speeds when only stern planes are utilised. Control performance slightly degrades when operating in neutrally buoyant condition. More overshoot and longer settling time are observed when changing depth at zero speed. This is because the PI-D is a linear controller that yields the best performance for a particular condition of that the controller is tuned. Despite this, the system could tolerate a fair level of net buoyancy variation, yielding satisfactory outcomes over the range of forward speeds.

The ability of the controller to respond to external variations, such as typical changes in buoyancy which could be observed in operation, are considered. The controller responds to this acceptably well.

By measuring the power utilised by the actuators, the impact of speed and net buoyancy have been recorded. The power consumption is significantly dependent on the magnitude of positive buoyancy. The vehicle hovering at zero speed consumes $52.05 \mathrm{~W}$ at $6 \mathrm{~N}$ net buoyancy whereas it consumes only $36.39 \mathrm{~W}$ at $1 \mathrm{~N}$. The buoyancy condition has less impact on this at high speeds.

\section{Acknowledgement}

This research is sponsored by the Faculty of International Maritime Studies, Kasetsart University, SriRacha Campus, Thailand.

The gratitude is also extended to Bertrand Malas and David Turner for their great support in using the Boldrewood Towing Tank, and Sophia M. Schillai for her assistance during the vehicle preparation and experiments.

\section{Appendix A. Free Ascent Test}

The AUV was programmed to dive to $2 \mathrm{~m}$ depth, at a zero propeller demand, and stayed steadily for $30 \mathrm{sec}$. Then, the two vertical thrusters were suddenly turned off, allowing the vehicle to float back to the water surface freely.

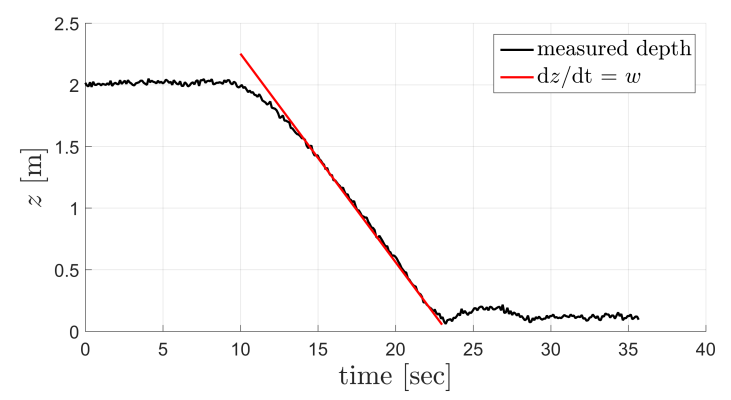

(a) depth response

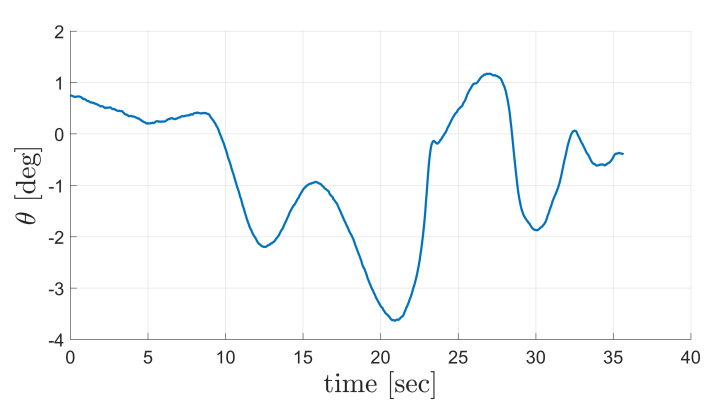

(b) pitch response

Figure A.21: Results from free ascent test.

The vehicle response is presented in figure A.21. Since a pitch angle variation is negligibly small, a heave velocity may be directly inferred from depth response.

Assuming the total force along $z$-axis is due to only the net buoyancy, i.e., $Z=W-B$. A quadratic heaving damping coefficient is determined as follows: $Z_{|w| w}=\frac{Z}{|w| w}$ 


\section{Appendix B. Model Parameters}

\begin{tabular}{|c|c|c|}
\hline Parameter & Value & Unit \\
\hline$m$ & 79.40 & $\mathrm{~kg}$ \\
\hline$\nabla$ & 0.08 & $\mathrm{~m}^{3}$ \\
\hline$W-B$ & -6 & $\mathrm{~N}$ \\
\hline$L_{A U V}$ & 1.96 & $\mathrm{~m}$ \\
\hline$I_{y y}$ & 35 & $\mathrm{~kg} \cdot \mathrm{m}^{2}$ \\
\hline$r_{b}$ & {$[0,0,0]$} & $\mathrm{m}$ \\
\hline$r_{g}$ & {$[0,0,0.06]$} & $\mathrm{m}$ \\
\hline$X_{\dot{u}}$ & -2.4 & $\mathrm{~kg}$ \\
\hline$Z_{\dot{w}}$ & -65.5 & $\mathrm{~kg}$ \\
\hline$M_{\dot{q}}$ & -14.17 & $\mathrm{~kg} \cdot \mathrm{m}^{2} / \mathrm{rad}$ \\
\hline$Z_{w}$ & $0.5 \rho L_{A U V}^{2} u(-28.5 \mathrm{e}-3)$ & $\mathrm{kg} / \mathrm{s}$ \\
\hline$Z_{q}$ & $0.5 \rho L_{A U V}^{3} u(-12.6 \mathrm{e}-3)$ & $\mathrm{kg} \cdot \mathrm{m} / \mathrm{rad} / \mathrm{s}$ \\
\hline$M_{w}$ & $0.5 \rho L_{A U V}^{3} u(-4.5 \mathrm{e}-3)$ & $\mathrm{kg} \cdot \mathrm{m} / \mathrm{s}$ \\
\hline$M_{q}$ & $0.5 \rho L_{A U V}^{4} u(-5.3 \mathrm{e}-3)$ & $\mathrm{kg} \cdot \mathrm{m}^{2} / \mathrm{rad} / \mathrm{s}$ \\
\hline$X_{|u| u}$ & -6.5 & $\mathrm{~kg} / \mathrm{m}$ \\
\hline$Z_{|w| w}$ & -183 & $\mathrm{~kg} / \mathrm{m}$ \\
\hline$Z_{|q| q}$ & 0 & $\mathrm{~kg} \cdot \mathrm{m} / \mathrm{rad}^{2}$ \\
\hline$M_{|w| w}$ & -59 & $\mathrm{~kg}$ \\
\hline$M_{|q| q}$ & -82 & $\mathrm{~kg} \cdot \mathrm{m}^{2} / \mathrm{rad}^{2}$ \\
\hline$K_{T_{0}, \text { prop }}$ & 0.0946 & - \\
\hline$D_{\text {prop }}$ & 0.305 & $\mathrm{~m}$ \\
\hline$c_{\text {prop }, 1}$ & 0.6999 & $\mathrm{n} / \mathrm{a}$ \\
\hline$c_{\text {prop }, 2}$ & 1.5205 & $\mathrm{n} / \mathrm{a}$ \\
\hline$K_{T, t h}$ & $1.2870 \mathrm{e}-4$ & - \\
\hline$D_{t h}$ & 0.07 & $\mathrm{~m}$ \\
\hline$w_{t}$ & 0.36 & - \\
\hline$t$ & 0.11 & - \\
\hline$L_{v}$ & 1.06 & $\mathrm{~m}$ \\
\hline$L_{v f}$ & 0.52 & $\mathrm{~m}$ \\
\hline$L_{v a}$ & -0.54 & $\mathrm{~m}$ \\
\hline$c_{t h, 1}$ & 0.35 & $\mathrm{n} / \mathrm{a}$ \\
\hline$c_{t h, 2}$ & 1.5 & $\mathrm{n} / \mathrm{a}$ \\
\hline$X_{u u \delta_{S} \delta_{S}}$ & -0.0036 & $\mathrm{~kg} / \mathrm{m} / \operatorname{deg}^{2}$ \\
\hline$Z_{u u \delta_{S}}$ & 0.3241 & $\mathrm{~kg} / \mathrm{m} / \mathrm{deg}$ \\
\hline$M_{u u \delta_{S}}$ & 0.3254 & $\mathrm{~kg} / \mathrm{deg}$ \\
\hline
\end{tabular}

\section{References}

[1] B. Allen, R. Stokey, T. Austin, N. Forrester, R. Goldsborough, M. Purcell, C. V. Alt, REMUS: a small, low cost AUV; system description, field trials and performance results, Oceans '97. MTS/IEEE Conference Proceedings 2.

[2] S. D. Mcphail, Autosub6000: A Deep Diving Long Range AUV, Journal of Bionic Engineering 6 (1) (2009) 55-62.

[3] R. Burcher, L. J. Rydill, Concepts in Submarine Design, Cambridge Ocean Technology Series, Cambridge University Press, 1995.
[4] D. Marco, A. Healey, Current developments in underwater vehicle control and navigation: the NPS ARIES AUV, OCEANS 2000 MTS/IEEE Conference and Exhibition. Conference Proceedings (Cat. No.00CH37158) 2.

[5] M. Dunbabin, J. Roberts, K. Usher, G. Winstanley, P. Corke, A Hybrid AUV Design for Shallow Water Reef Navigation, in: Proceedings of the 2005 IEEE International Conference on Robotics and Automation, IEEE, 2005, pp. 2105-2110.

[6] G. Packard, R. Stokey, R. Christenson, F. Jaffre, M. Purcell, R. Littlefield, Hull inspection and confined area search capabilities of REMUS autonomous underwater vehicle, Oceans 2010 (2010) 1-4.

[7] D. Ribas, P. Ridao, A. Turetta, C. Melchiorri, G. Palli, J. J. Fernandez, P. J. Sanz, I-AUV Mechatronics Integration for the TRIDENT FP7 Project, IEEE/ASME Transactions on Mechatronics 20 (5) (2015) 2583-2592.

[8] B. Jalving, The NDRE-AUV flight control system, IEEE Journal of Oceanic Engineering 19 (4) (1994) 497-501.

[9] S. B. Williams, P. Newman, D. Gamini, J. Rosenblatt, H. Durrant-whyte, A decoupled, distributed AUV control architecture, in: Proc. of 31st International Symposium on Robotics, 2000, pp. 246-251.

[10] R. McEwen, K. Streitlien, Modeling and control of a variable-length auv, Proc 12th UUST (2001) 1-42.

[11] Y. H. Eng, H. G. Soon, M. Chitre, Depth control of an autonomous underwater vehicle, STARFISH, in: OCEANS'10 IEEE SYDNEY, IEEE, 2010, pp. 1-6.

[12] Y. H. Eng, M. Chitre, K. M. Ng, K. M. Teo, Minimum Speed Seeking Control for Nonhovering Autonomous Underwater Vehicles, Journal of Field Robotics 7 (PART 1) (2015) 1-28.

[13] O. Härkegård, S. T. Glad, Resolving actuator redundancyoptimal control vs. control allocation, Automatica 41 (1) (2005) 137-144.

[14] T. I. Fossen, T. Johansen, A Survey of Control Allocation Methods for Ships and Underwater Vehicles, in: 2006 14th Mediterranean Conference on Control and Automation, IEEE, 2006, pp. 1-6.

[15] M. Kokegei, F. He, K. Sammut, Fully Coupled 6 Degree-of-Freedom Control of an Over-Actuated Autonomous Underwater Vehicle, in: N. Cruz (Ed.), Autonomous Underwater Vehicles, InTech, 2011, pp. 147-170.

[16] F. Dukan, M. Ludvigsen, A. J. Sørensen, Dynamic positioning system for a small size ROV with experimental results, OCEANS 2011 IEEE - Spain.

[17] S. Jin, J. Kim, J. Kim, T. Seo, Six-Degree-of-Freedom Hovering Control of an Underwater Robotic Platform With Four Tilting Thrusters via Selective Switching Control, in: Mechatronics, IEEE/ASME Transactions on , vol.PP, no.99, IEEE, 2014, pp. 1-9.

[18] A. Alvarez, A. Caffaz, A. Caiti, G. Casalino, 
L. Gualdesi, A. Turetta, R. Viviani, Fòlaga: A lowcost autonomous underwater vehicle combining glider and AUV capabilities, Ocean Engineering 36 (1) (2009) 24-38.

[19] G. Indiveri, A. Malerba, Complementary control for robots with actuator redundancy: an underwater vehicle application, Robotica (May) (2015) 1-18.

[20] C. Nickell, C. Woolsey, D. Stilwell, A Low-Speed Control Module for a Streamlined AUV, in: Proceedings of OCEANS 2005 MTS/IEEE, Vol. 2005, IEEE, 2005, pp. $1-6$.

[21] B. Thornton, T. Ura, Y. Nose, S. Turnock, ZeroG Class Underwater Robots: Unrestricted Attitude Control Using Control Moment Gyros, IEEE Journal of Oceanic Engineering 32 (3) (2007) 565-583.

[22] M. Breivik, T. I. Fossen, A unified control concept for autonomous underwater vehicles, in: 2006 American Control Conference, IEEE, 2006, p. 7 pp.

[23] A. Palmer, G. Hearn, P. Stevenson, A theoretical approach to facilitating transition phase motion in a positively buoyant autonomous underwater vehicle, in: Transactions of RINA, Part A3 - International Journal of Maritime Engineering (IJME), Vol. 151, 2009, pp. 1-16.

[24] L. V. Steenson, S. R. Turnock, A. B. Phillips, C. Harris, M.E. Furlong, E. Rogers, L. Wang, K. Bodles, D.W. Evans, M. E. Furlong, D. W. Evans, Model predictive control of a hybrid autonomous underwater vehicle with experimental verification, Proceedings of the Institution of Mechanical Engineers, Part M: Journal of Engineering for the Maritime Environment 228 (2) (2014) 166-179.

[25] P. Jantapremjit, P. A. Wilson, Guidance-Control Based Path Following for Homing and Docking using an Autonomous Underwater Vehicle, OCEANS 2008 - MTS/IEEE Kobe Techno-Ocean (1) (2008) 1-6.

[26] T. I. Fossen, Handbook of Marine Craft Hydrodynamics and Motion Control, John Wiley \& Sons, Ltd, Chichester, UK, 2011.

[27] A. Levant, A. Pridor, R. Gitizadeh, I. Yaesh, J. Z. Ben-Asher, Aircraft Pitch Control via Second-Order Sliding Technique, Journal of Guidance, Control, and Dynamics 23 (4) (2000) 586-594.

[28] J. E. Ruiz-Duarte, A. G. Loukianov, Higher Order Sliding Mode Control for Autonomous Underwater Vehicles in the Diving Plane, IFAC-PapersOnLine 48 (16) (2015) 49-54.

[29] A. B. Phillips, L. V. Steenson, J. Liu, Delphin2: An over actuated autonomous underwater vehicle for manoeuvring research, Transactions of the Royal Institution of Naval Architects, Part A Engineering 151 (0092-8674) (2013) 1-11.

[30] G. Griffiths, Technology and applications of autonomous underwater vehicles, 2002.

[31] L. V. Steenson, Experimentally Verified Model Predictive Control of a Hover-Capable AUV, Ph.D. the- sis, University of Southampton (2013).

[32] SNAME, Nomenclature for treating the motion of a submerged body through a fluid, Tech. rep., The Society of Naval Architects and Marine Engineers (1952).

[33] N. I. Kimber, W. B. Marshfield, Design and testing of control surfaces for the autosub demonstrator test vehicle, Tech. rep., Institute of Oceanographic Sciences (1993).

[34] J. Norris, Wind Tunnel Testing of The Delphin2 AUV, Master's thesis, University of Southampton, Southampton, UK (2012).

[35] A. Palmer, Analysis of the propulsion and manoeuvring characteristics of survey-style AUVs and the development of a multi-purpose AUV, Ph.D. thesis, University of Southampton (2009).

[36] A. Palmer, G. E. Hearn, P. Stevenson, Experimental Testing of an Autonomous Underwater Vehicle with Tunnel Thrusters, in: First International Symposium on Marine Propulsion, 2009, pp. 1-6.

[37] A. F. Molland, S. R. Turnock, D. A. Hudson, Ship resistance and propulsion: practical estimation of propulsive power, Cambridge university press, 2011.

[38] APC Performance Data Files, APC Performance Data Files, last accessed on Jan 09, 2016. (2016). Available from: URL : http: //apcserve.w20.wh-2. com/v/PERFILES_WEB/PER3_12x6EP(F2B). dat

[39] A. Palmer, G. Hearn, P. Stevenson, Modelling tunnel thrusters for autonomous underwater vehicles, in: Navigation, Guidance and Control of Underwater Vehicles (NGCUV'08), International Federation of $\mathrm{Au}$ tomatic Control (IFAC), 2008, pp. 1-6.

[40] K. Ogata, Modern Control Engineering, 5th Edition, Prentice Hall, 2009.

[41] L. V. Steenson, A. B. Phillips, M. E. Furlong, E. Rogers, S. R. Turnock, The performance of vertical tunnel thrusters on an autonomous underwater vehicle operating near the free surface in waves, in: Second International Symposium on Marine Propulsors, 2011, pp. 1-8.

[42] A. B. Phillips, M. Haroutunian, S. K. Man, A. J. Murphy, S. W. Boyd, J. I. R. Blake, G. Griffiths, Nature in engineering for monitoring the oceans: comparison of the energetic costs of marine animals and AUVs, in: G. N. Roberts, R. Sutton (Eds.), Further Advances in Unmanned Marine Vehicles, Institution of Engineering and Technology, pp. 373-405.

[43] A. B. Phillips, M. Haroutunian, A. J. Murphy, S. W. Boyd, J. I. R. Blake, G. Griffiths, Understanding the power requirements of autonomous underwater systems, Part I: An analytical model for optimum swimming speeds and cost of transport, Ocean Engineering (January 2016). 


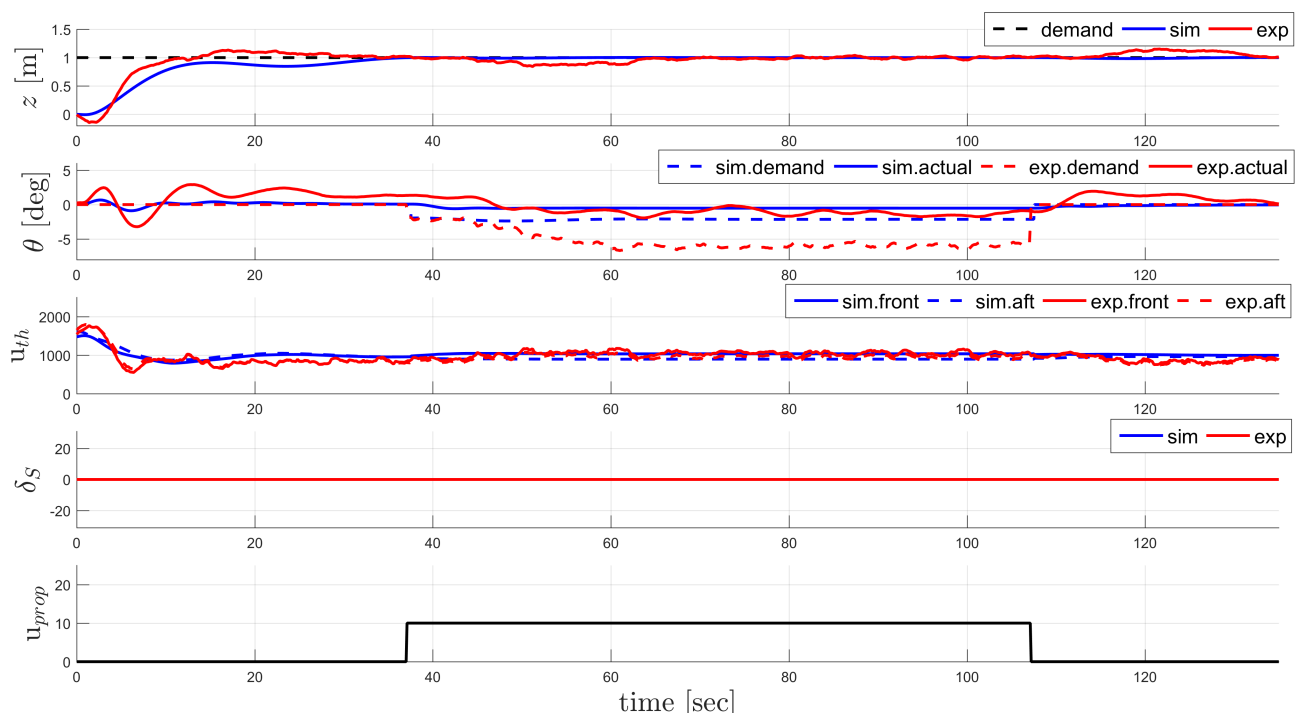

(a) low-speed case $\left(\mathrm{u}_{\text {prop }}=10\right)$

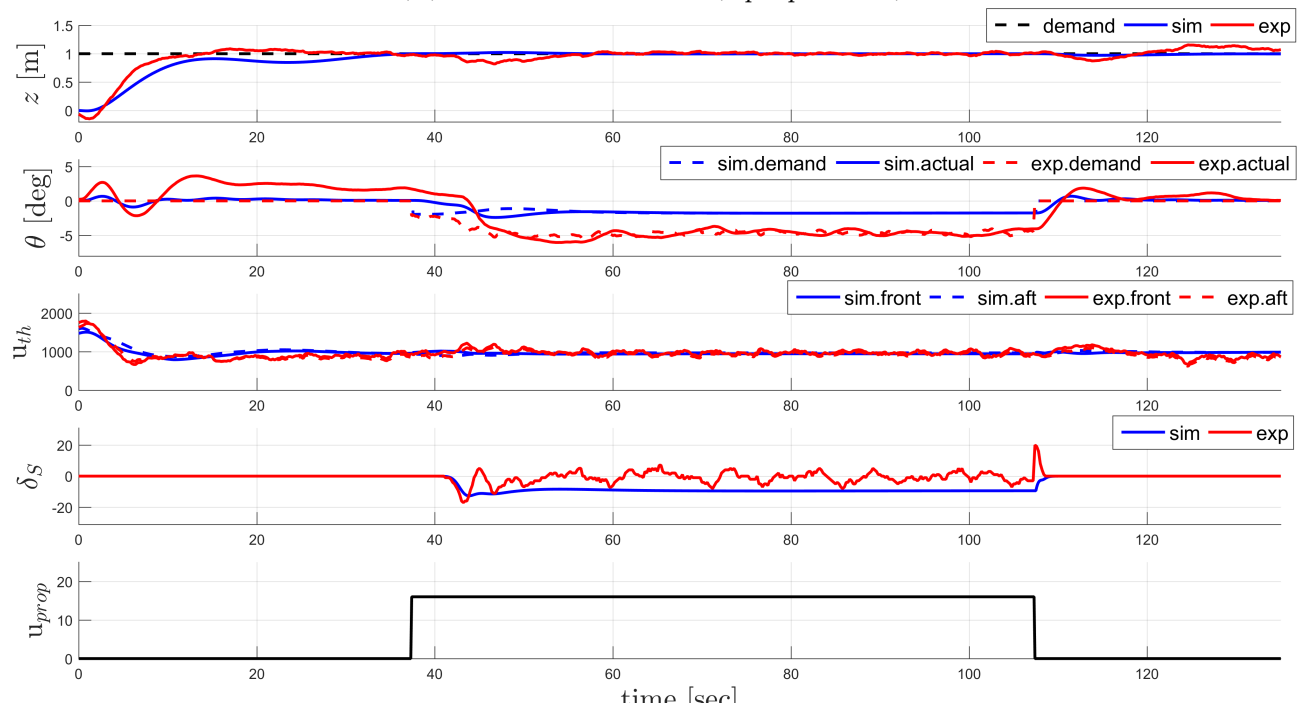

(b) mid-speed case $\left(\mathrm{u}_{\text {prop }}=16\right)$

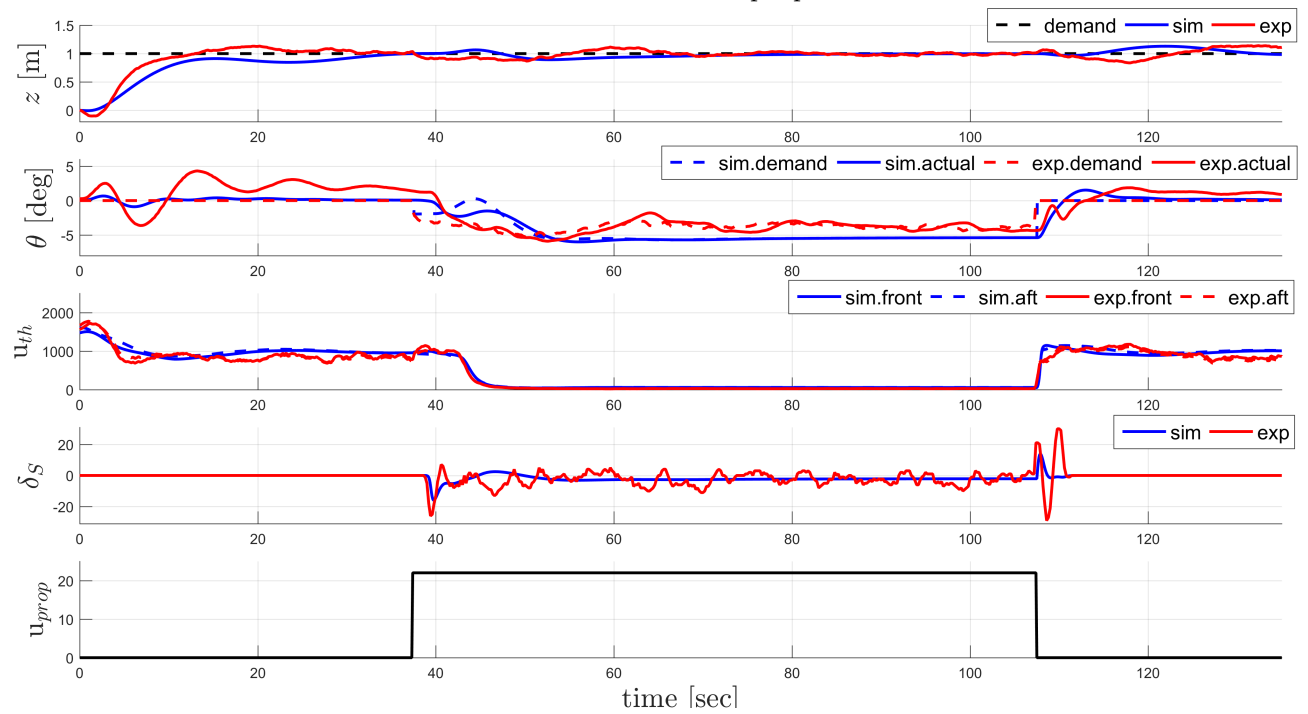

(c) high-speed case $\left(u_{\text {prop }}=22\right)$

Figure B.22: Flight-style operation at speeds with a speed transition. 


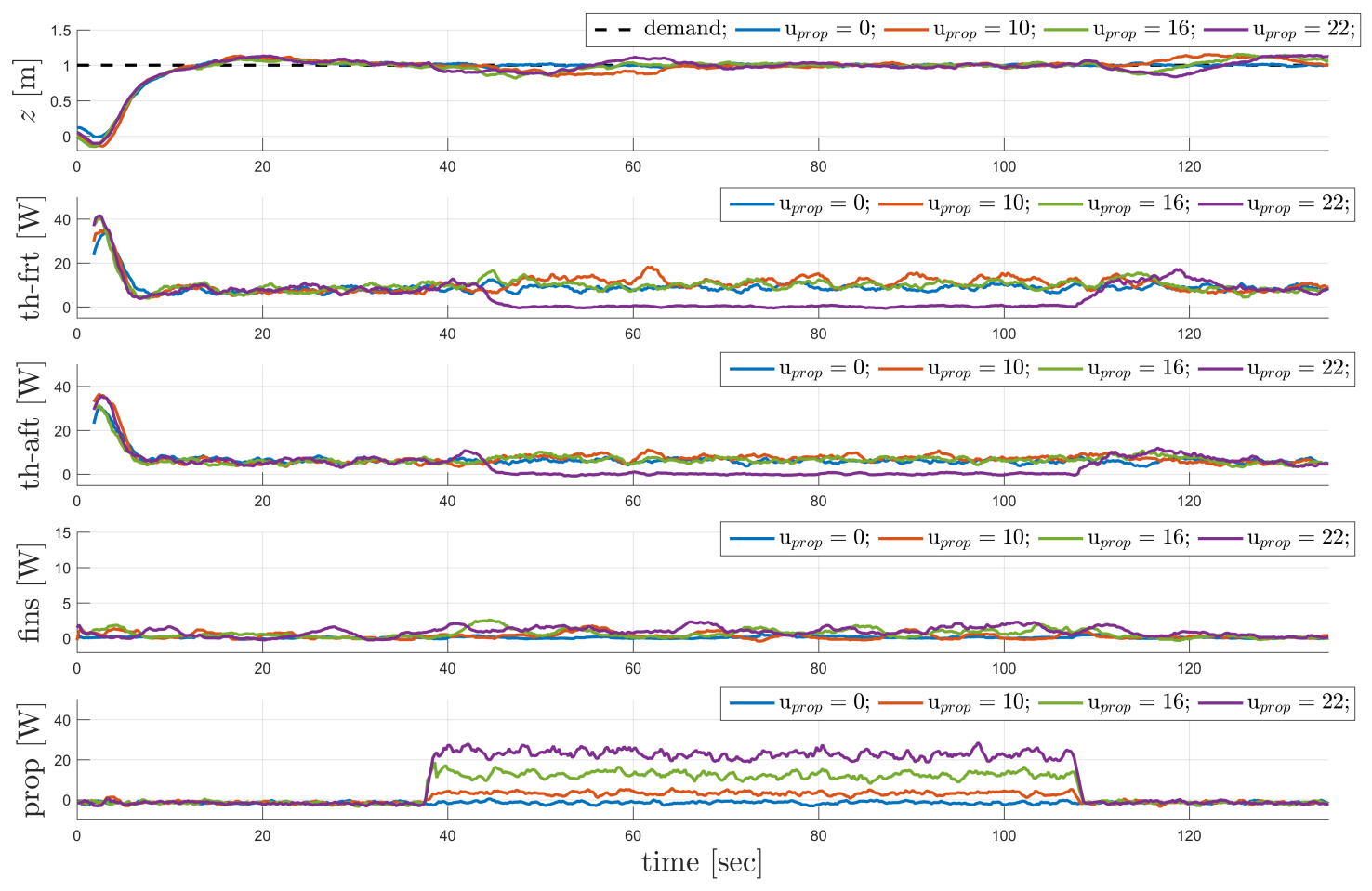

(a) $+6 \mathrm{~N}$ buoyant

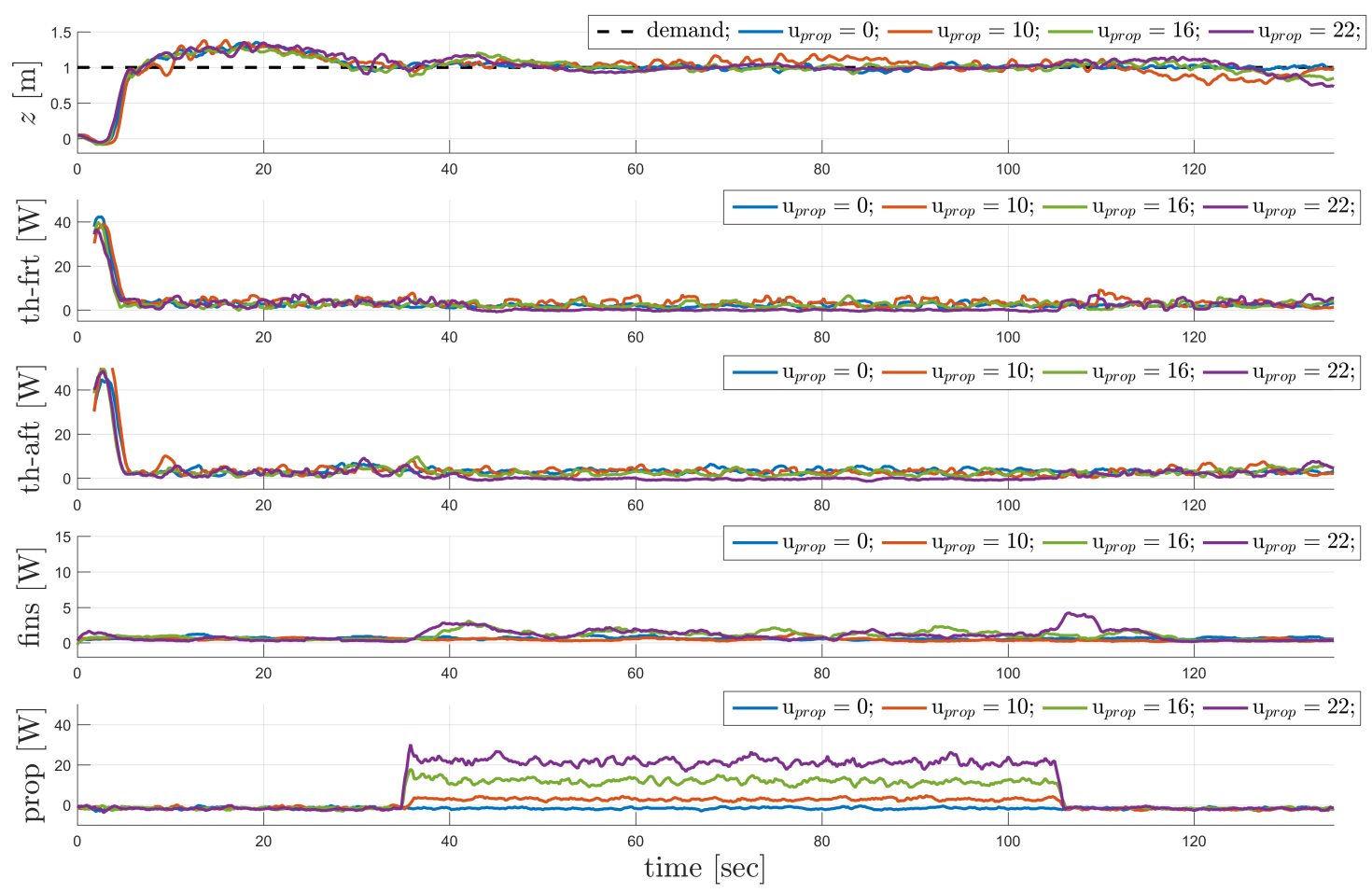

(b) $+1 \mathrm{~N}$ buoyant

Figure B.23: Experimental results show power consumption on actuators when travelling at different speeds and buoyancy conditions. 